\title{
WestVirginiaUniversity
}

THE RESEARCH REPOSITORY @ WVU

Graduate Theses, Dissertations, and Problem Reports

2016

\section{Heat Transfer Prediction for Single Droplet Impacts on a wetted Surface}

Venkata Giriteja Punnamaraju

Follow this and additional works at: https://researchrepository.wvu.edu/etd

\section{Recommended Citation}

Punnamaraju, Venkata Giriteja, "Heat Transfer Prediction for Single Droplet Impacts on a wetted Surface" (2016). Graduate Theses, Dissertations, and Problem Reports. 6458.

https://researchrepository.wvu.edu/etd/6458

This Thesis is protected by copyright and/or related rights. It has been brought to you by the The Research Repository @ WVU with permission from the rights-holder(s). You are free to use this Thesis in any way that is permitted by the copyright and related rights legislation that applies to your use. For other uses you must obtain permission from the rights-holder(s) directly, unless additional rights are indicated by a Creative Commons license in the record and/ or on the work itself. This Thesis has been accepted for inclusion in WVU Graduate Theses, Dissertations, and Problem Reports collection by an authorized administrator of The Research Repository @ WVU. For more information, please contact researchrepository@mail.wvu.edu. 


\title{
Heat Transfer Prediction for Single Droplet Impacts on a wetted Surface
}

\author{
Venkata Giriteja Punnamaraju
}

Thesis submitted to the

Benjamin M. Statler College of Engineering and Mineral Resources

at West Virginia University

in partial fulfillment of the requirements for the degree

\author{
Master of Science \\ in \\ Mechanical Engineering \\ John M. Kuhlman, Ph.D., Chair \\ Patrick Browning, Ph.D \\ Donald D. Gray, Ph.D \\ Department of Mechanical and Aerospace Engineering \\ Morgantown, West Virginia \\ 2016
}

Keywords: Spray Cooling, Energy Equation, CFD, OpenFOAM, VOF

Copyright 2016 Venkata Giriteja Punnamaraju 


\title{
Heat Transfer Prediction for Single Droplet Impacts on a wetted Surface
}

\author{
Venkata Giriteja Punnamaraju \\ Thesis submitted to the
}

Benjamin M. Statler College of Engineering and Mineral Resources

at West Virginia University

in partial fulfillment of the requirements for the degree

\author{
Master of Science \\ in \\ Mechanical Engineering \\ Department of Mechanical and Aerospace Engineering \\ APPROVAL OF THE EXAMINING COMMITTEE
}

Patrick Browning, Ph. D.

Donald D. Gray, Ph. D.

Date

John M. Kuhlman, Ph. D, Chair 


\section{Abstract \\ Heat Transfer Prediction for Single Droplet Impacts \\ Venkata Giriteja Punnamaraju}

The decrease in sizes of semiconductors and microelectronic devices also decreases their ability to eject energy in the form of heat due to their small surface areas, which can lead to their failure. Spray cooling is one of the better high heat flux cooling techniques.

As an initial step, the impact of a single liquid drop on a thin liquid film has been simulated and studied, taking the energy equation into consideration. All the cases have been simulated in 3D with an open source CFD package OpenFOAM using the Volume of Fluid (VOF) model. The post-processing of the data was done in ParaView and MATLAB to obtain the predicted heat flux in the droplet impact cavity, the film thickness beneath the cavity and the sub-cavity liquid volume as functions of time. The sub-cavity film thickness and the liquid volume results are compared with the experiments. The cavity heat flux is predicted to be significantly higher than heat flux predictions outside the cavity over much of the cavity lifetime. 


\section{Table of Contents}



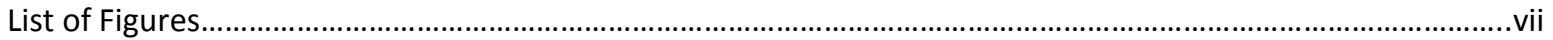

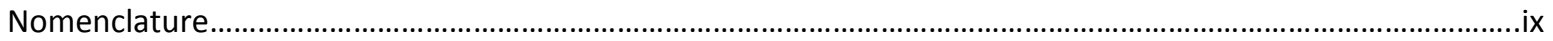

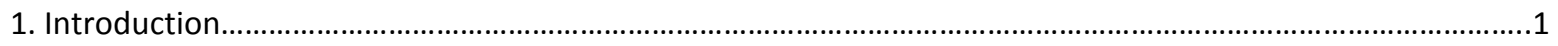

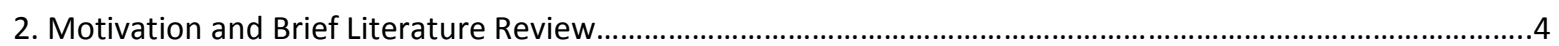



3.1 VOF model in OpenFOAM.............................................................................................................

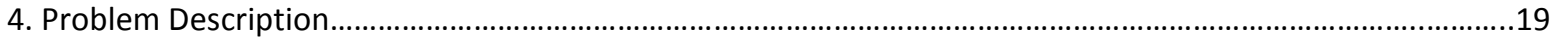



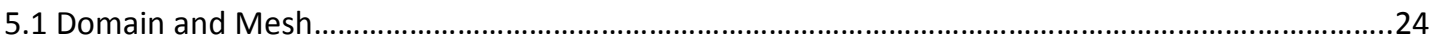

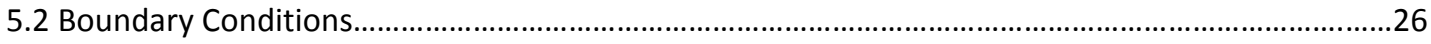

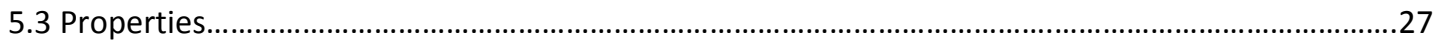

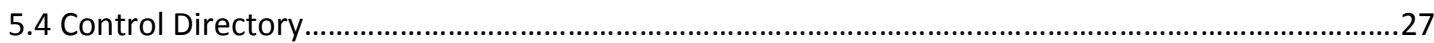



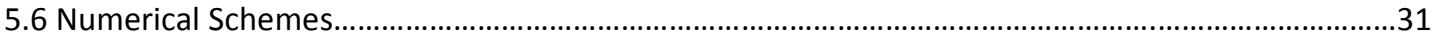

5.7 Setting the Fields........................................................................................................................

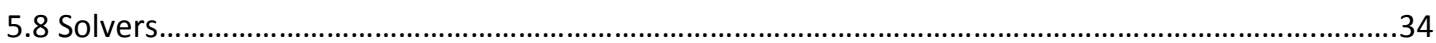



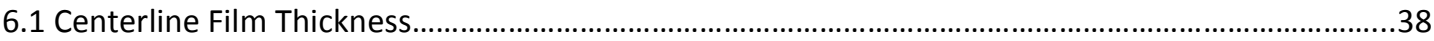



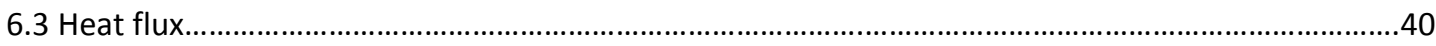



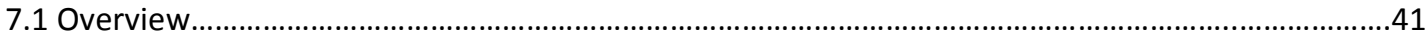

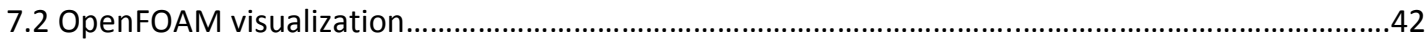

7.3 Comparison of Centerline Film Thickness........................................................................................

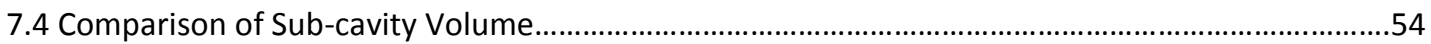

7.5 Average heat Flux..........................................................................................................................

8. Conclusions and Future Work........................................................................................................................64

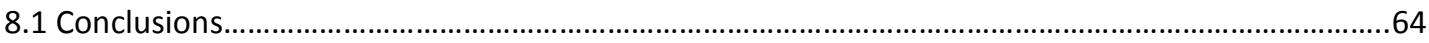

8.2 Future Work

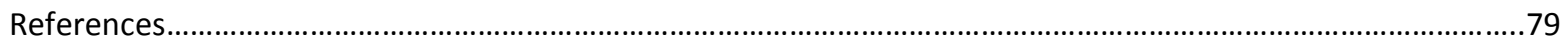




\section{Acknowledgement}

I thank Dr. John M. Kuhlman for identifying me as one of the students who could do this "Spray Cooling" project. He guided me with great patience, and he was with me even during my other issues in the university. He always gave me time during his busy schedule which also motivated me to work had. I really feel privileged to be associated with him and to work under him.

I would also like to thank Dr. Donald D. Gray and Dr. Patrick Browning for making my thesis neat and complete without any mistakes.

I specially thank National Science Foundation EPSCoR, the state of West Virginia (WVEPSCoR) and WVU for crucial resource for accomplishing this work. Super Computing System (Mountaineer) is funded by them. I should also thank Nathan Gregg for his patience and support in answering my basic questions initially.

I should really thank József Nagy, Johannes Kepler University, Linz, Austria on this note. He really helped me when I was stuck and didn't know what do. Even though we are not on the same timeline, he used to solve my problems and respond almost immediately.

I take pleasure in thanking Mr. Krishna Teja Medam who helped me a lot with the basics of OpenFOAM even during his tight schedule. I really thank Ajay Krishna Teja Kavuri, my roommate, as he helped regarding 
all the computer and software issues that I've faced. I thank Mr. Sai

Satish Guda to help me solve technical problems in CFD.

I thank Mr. Gennaro Campitelli and Mr. Nicholas Lee Hillen who helped with their inputs.

I especially thank my parents for sending me so far and to help me achieving my goals. They backed me whenever I was down and they guided me whenever I was out of focus and concentration. I also thank all my friends, especially Lahari Vazrala and Rupindrani Aila in Morgantown for helping me in some or the other way. 


\section{List of figures}

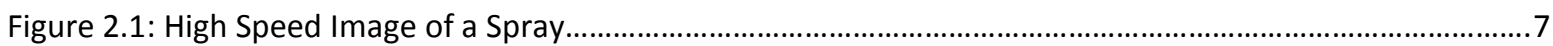



Figure 2.3: Impact of a droplet on a liquid film and its components...................................................................10

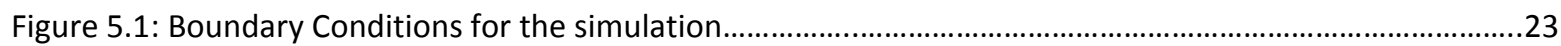







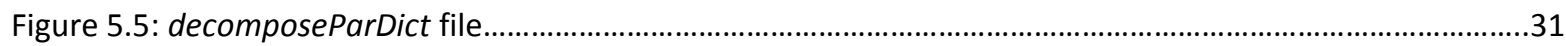

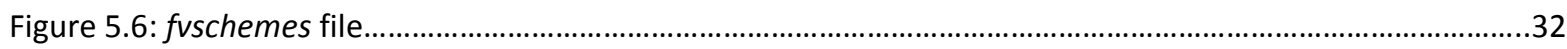

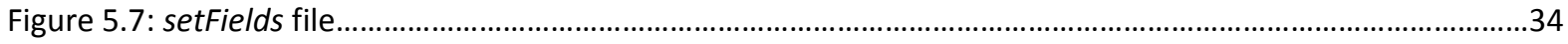



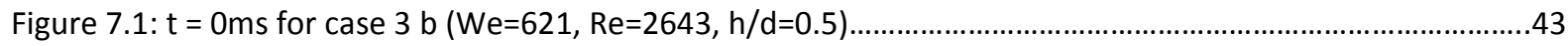

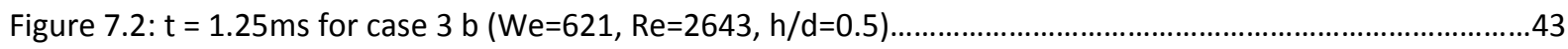



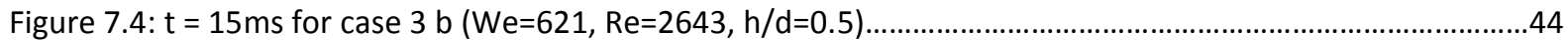

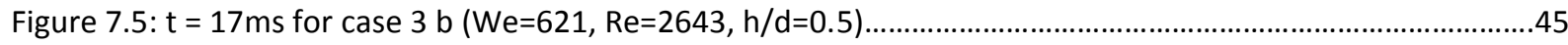



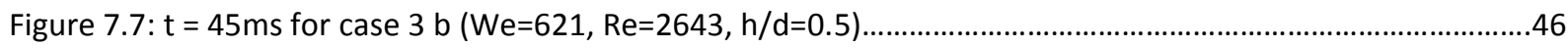



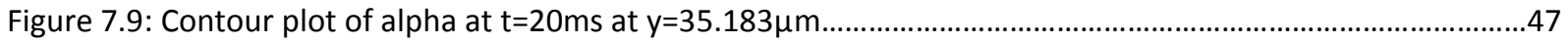

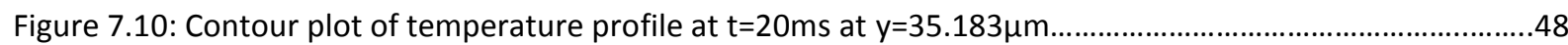



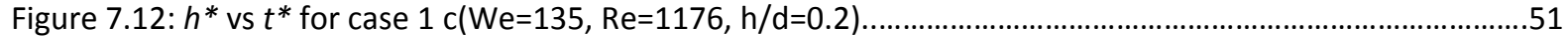

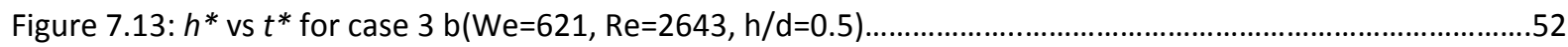

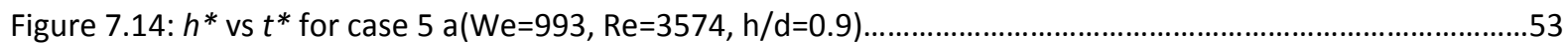

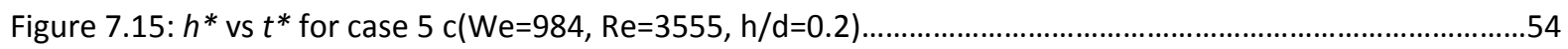



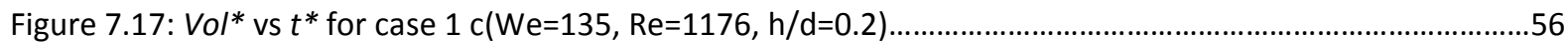










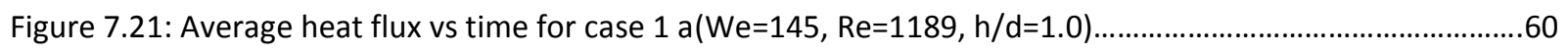





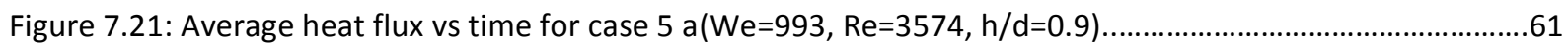

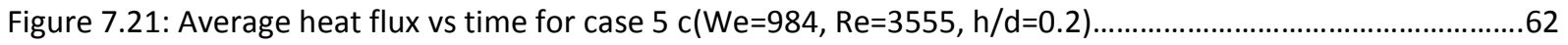




\section{List of Tables}

Table 4.1: List of all cases with the values of the parameters.....................................................................21 


\begin{tabular}{|c|c|}
\hline 2D & Two Dimensional \\
\hline 3D & Three Dimensional \\
\hline CFD & Computational Fluid Dynamics \\
\hline$d$ & diameter of the impinging droplet \\
\hline EPSCoR & Experimental Program to simulate Competitive Research \\
\hline $\mathrm{Fr}$ & Froude number \\
\hline h & $\begin{array}{l}\text { Height of the liquid above the bottom wall at the impact } \\
\text { center }\end{array}$ \\
\hline$h_{0}$ & Initial static liquid layer above the bottom wall \\
\hline$h_{0} *$ & Normalized static liquid layer height $\left(h_{0} / d\right)$ \\
\hline$h^{*}$ & Normalized height of the liquid $(\mathrm{h} / \mathrm{d})$ \\
\hline h_min & $\begin{array}{l}\text { Average height of the liquid at the impact center over } \\
\text { time }\end{array}$ \\
\hline k & Thermal Conductivity \\
\hline MC Model & Monte-Carlo Model \\
\hline $\mathrm{Oh}$ & Ohnesorge number \\
\hline Q & Volumetric Flow Rate \\
\hline$q$ & Temperature heat Flux \\
\hline $\operatorname{Re}$ & Reynolds number \\
\hline $\mathrm{T}$ & Temperature \\
\hline $\mathrm{t}$ & Time taken \\
\hline$t^{*}$ & $=\mathrm{t} \frac{V e l}{d}$ \\
\hline
\end{tabular}


VOF

$V_{\text {drop }}$

$V_{\text {sub-cavity }}$

Vel

$\mathrm{V}^{*}$

WVEPSCOR

We
Volume of Fluids

Volume of the droplet

Sub-cavity Volume

Velocity of the impinging Droplet

$=($ Vsub - cavity $) /$ Vdrop

West Virginia Experimental Program to Stimulate

Competitive Research

Weber number 


\section{Introduction:}

The sizes of the microelectronic components and semiconductors are decreasing which increases the necessity for high heat flux cooling techniques. The wasted energy which is produced from the components is released in the form of heat energy. If this thermal energy is not removed or not treated properly, it eventually leads to the failure of the component. Since the size of the components is smaller, the surface area from where it releases heat is also small. So there is a need for high heat flux cooling techniques. Of the many heat flux cooling techniques, the following have gained great prominence: micro-channel, jet-impingement and spray cooling. This thesis concentrates mainly on spray cooling. The advantages of spray cooling are i) high-flux heat dissipation, ii) low and fairly uniform temperature and iii) the ability to provide cooling for a considerably large surface area with a single nozzle.

In spray cooling, liquid at local environment temperature is sprayed on the component, the waste heat transfers from the component to the liquid due to conduction and convection. The liquid sometimes reaches its boiling point and evaporates, eventually removing the heat produced by the component. Simulating spray and its interaction with hot surface requires High-Performance 
Computation. As a beginning step, a single droplet from spray is analyzed in this work using Computational Fluid Dynamics (CFD) based on the expected ranges of single droplet Reynolds number, Weber number, and Froude number. Film thickness and sub-cavity volume are calculated for cases with the energy equation implemented in the CFD model. 3D simulations were made using OpenFOAM4:0:0. All the simulations were performed using the Volume of Fluid (VOF) computational model. This model gives better interface capture than the level set method, as the cases that are simulated here require precise liquid thickness height to calculate the sub-cavity volume. This sub-cavity volume is needed to predict the heat required to evaporate the sub-cavity volume of liquid, which eventually cools the heated surface.

Also computed from the VOF model is the local wall heat flux in the sub-cavity region. This information is needed in order to quantify the level of enhancement that will occur for the local transient conduction in the droplet impact cavity.

The main aim of this thesis is to find the behavior of sub-cavity liquid volume and sub-cavity liquid temperature with respect to time, which eventually gives us the local sub-cavity heat flux required to evaporate this sub-cavity volume of liquid 
from the heated surface, by which the temperature of the heated surface is controlled. 


\section{Motivation and Brief Literature Review}

Spray cooling is one of the most effective heat transfer techniques. The electrical components reject heat as the by-product when they are functioning across a minuscule surface area, which when not cooled properly can lead to the failure of the component. This is where spray cooling helps in controlling the temperature of the component by removing the excess heat from the component. When a cooler liquid is sprayed on the hot surface, the liquid, which is below the Leidenfrost point gets accumulated on the surface. The accumulated liquid layer absorbs heat from the surface. When a surface reaches a certain liquid layer thickness, any further spraying leads to the formation of cavities in the liquid layer formed. Heat transfer takes place when a relatively cool droplet hits the residual liquid layer. There will be a high local transient heat flux beneath the cavity. These cavities have a very thin layer of liquid where the sub-cavity liquid (beneath the liquid cavity) heats up quickly and may start boiling. These cavity sites also initially act as active sites for nucleate boiling, partly due to entrapped vapor or gas due to droplet impact (Campbell et al., 1999), which eventually evaporates the liquid. The evaporation of the sub-cavity liquid then leads to an increase of heat transfer from the surface in the cavity, unless the cavity fills in with the liquid from the 
surrounding thicker liquid layer prior to dry out. The heat transfer takes place when a liquid is heated, boiled and evaporated.

The characteristics of liquid droplets impacting a thin liquid film have a great importance in engineering and technological applications such as spray cooling, spray coating, spray painting and ink jet printing. Spray cooling is widely used in IC engines, space technology, defense, food industry, fuel sprays in gas turbines, medical industries, HVAC industries, electronics, agricultural industries and much more. The high heat fluxes achievable in spray cooling can keep the component in the required temperature range. Any failures caused due to the elevated component temperature can be avoided. Heat flux reaching up to 700 $\mathrm{W} / \mathrm{cm}^{2}$ was claimed to be possible by the NSA at Supercomputing Research (2009). Using a Micromachined spray head, heat ejection of $250 \mathrm{~W} / \mathrm{cm}^{2}$ was achieved. Spray cooling experiments by Lin and Ponnappan(2003) demonstrated heat fluxes of $500 \mathrm{~W} / \mathrm{cm}^{2}$ before the onset of critical heat flux (CHF) using water as their coolant. They also achieved heat fluxes of $100 \mathrm{~W} / \mathrm{cm}^{2}$ at relatively low superheats ( $\left.T_{\text {wall }}-T_{\text {sat }}\right)$ of approximately $12 \mathrm{~K}$ using water as the coolant.

There are two main methods used in predicting or determining the performance of spray cooling systems: physically performing the experiment, or 
simulating using Computational Fluid Dynamics (CFD) and an analytical approach. However, issues in the required resources and time are limitations for all the methods. The use of CFD in completely simulating the spray would require an innumerous number of computational resources and a lot of time due to the complexity of the spray. Krietzer(2010) and Kuhlman et al.(2011) state that the droplet impact density fluxes may cross even $10^{6}$ drops $/\left(\mathrm{s} . \mathrm{cm}^{2}\right)$. These kind of sprays are extremely difficult to simulate. Figure 2.1 shows an example from the high-speed video image from the experiments with a relatively high volumetric flow rate of $Q=1 \times 10^{-5} \mathrm{~m}^{3} / \mathrm{s}$ sprayed onto a $16 \mathrm{~mm}$ diameter impact surface. These images were taken during the cavity formation and its lifetime. 


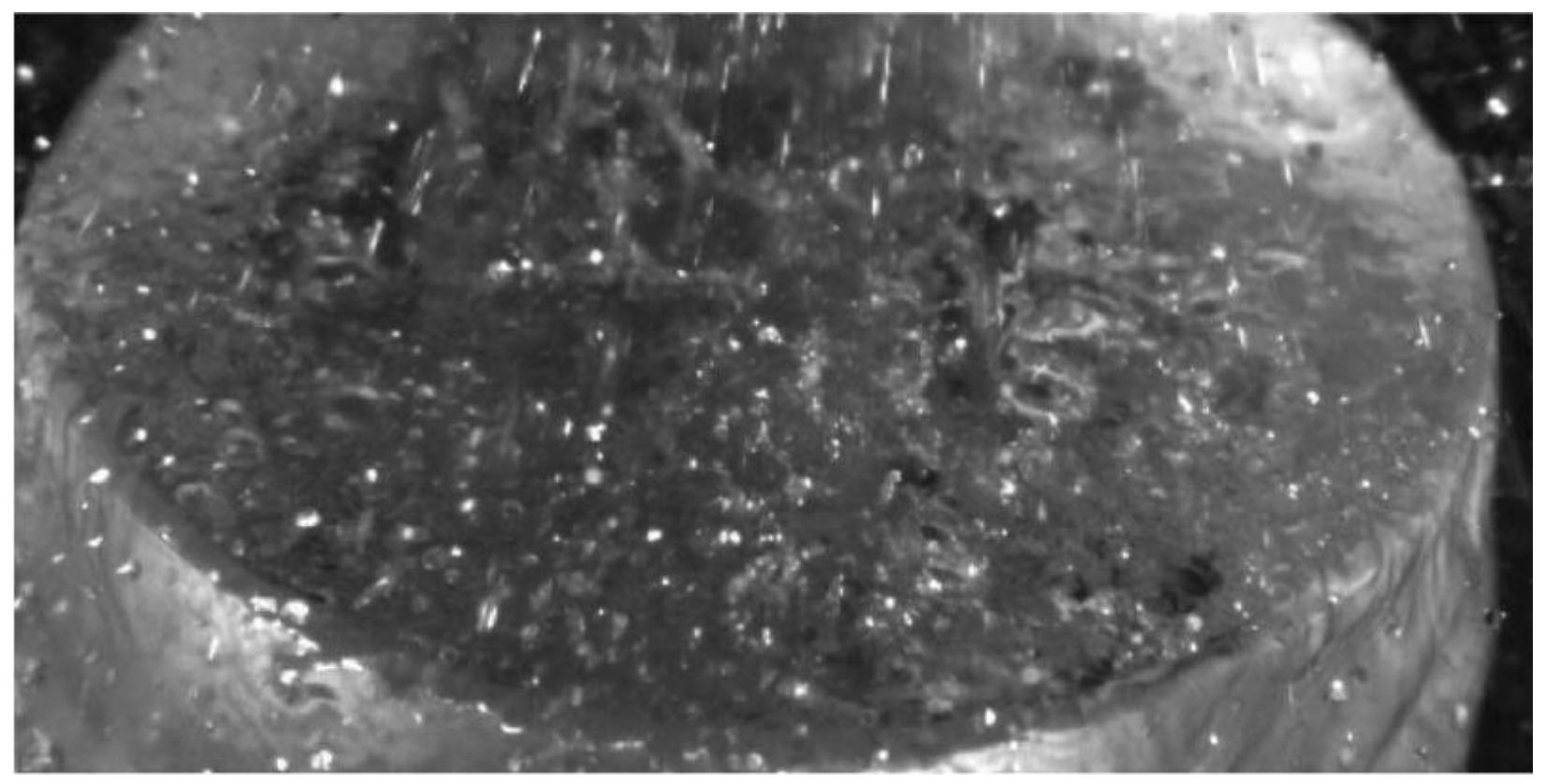

Fig. 2.1 High Speed Image of a spray (Kreitzer,2010)

To develop the practical model of spray, a preliminary Monte-Carlo (MC) model of spray simulation has been developed to predict the characteristics of spray. The initial MC model developed by Krietzer (2010), is still under development, with the ultimate goal of predicting the heat flux of a given nozzle flow conditions accurately for smooth heater surface properties and with the temperatures below the Leidenfrost point in reasonable computational times.

The MC model is a statistical approach to model the flow and heat transfer process without actually solving the differential equations and is effective in predicting the heat transfer and simulating the fluid flow. The initial MC model 
developed by Krietzer (2010) incorporate empirical correlations developed from his experiments and CFD simulations. It still lacks all of the necessary physics to be accurate. The main focus of the present thesis is on the volume of the liquid below the droplet impact, called as sub-cavity volume hereafter and the temperature prediction of the sub-cavity liquid after the droplet impact. Their importance will be discussed more completely later on.

Due to the effects of surface tension, impact of neighboring droplets, diameter of the droplet, the height from which it is dropped, the temperature of the liquid layer, and the effects of gravity, the cavity fills in quickly, and can form a 'Worthington jet' (Fig. 2.2), that raises the surface to a maximum height due to the effects of surface tension, gravity and velocity of the droplet at the time of impact. 


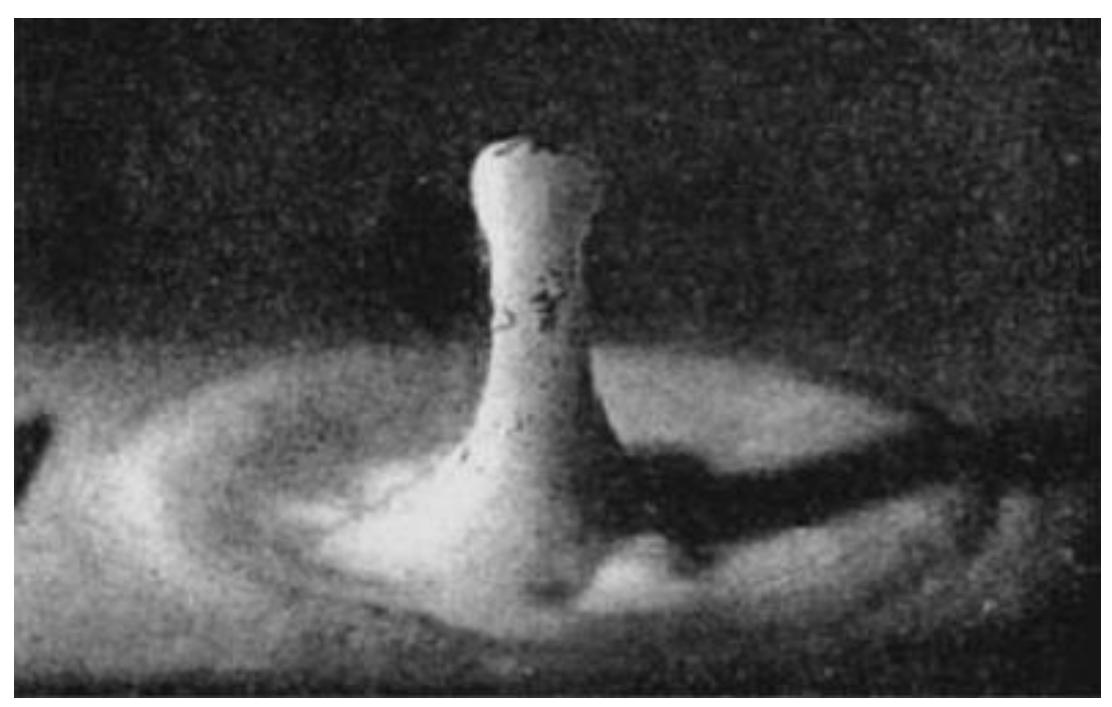

Fig. 2.2 Worthington jet formation of a milk drop

Kuhlman et al., (2007), argue that sub-cavity volume plays a crucial role in the heat transfer processes like onset and boiling of liquid and drying out the cavity. The cavity formed has a thin liquid film beneath the cavity for a given initial drop liquid and initially heated film liquid. Local heat fluxes are high due to the close proximity of cooler liquid with the surface. The expected sub-cavity volumes are expected to experience rapid heating and onset of boiling due to higher heat fluxes but will be vulnerable leading to drying out the cavity before it is refilled by the effects of surface tension and gravity or by neighboring droplet impacts.

The present thesis discusses the impact of the single droplet on a static liquid film at a certain initial temperature, with various values of the static liquid film thickness to droplet diameter $(\mathrm{h} / \mathrm{d})$ ratio and Weber numbers. In the present research of spray cooling, the central aim is to calculate the amount of heat 
transfer that is taking place. Yarin (2006) investigated the individual droplet impact, formation of the crown and the criteria for the onset of secondary splash and Worthington jet formation, and the effects of droplet impact on both liquid films and dry surfaces. Fig. 2.2 shows the impact of a milk droplet on a thin liquid film and formation of the crown. Figure 2.3 shows an example of a droplet impact on a thin liquid film with its major features defined.

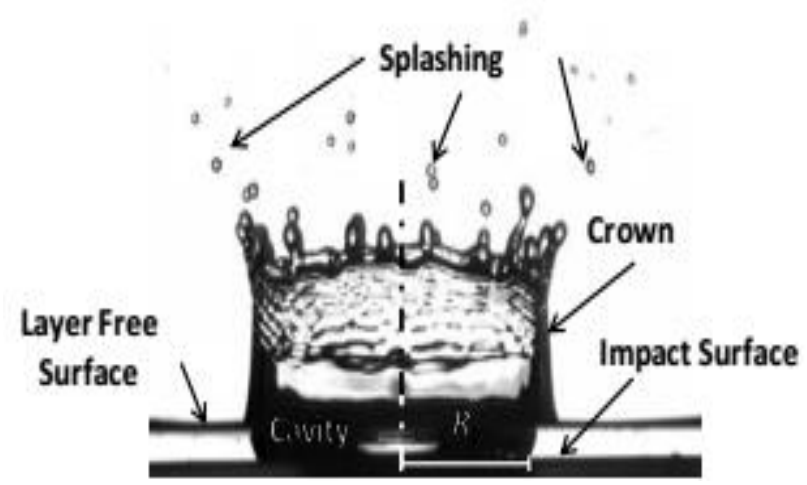

Fig. 2.3 Impact of a droplet on a liquid film and its components (Hillen et al. 2013)

Dimensionless numbers have been identified to match the single droplet experimental results or CFD simulation results to that of corresponding spray 
results. The most important of these is the Weber number and is defined as follows:

$$
\mathrm{We}=\frac{\rho v^{2} d}{\sigma}
$$

It is often useful in analyzing fluid flows where there is an interface between two different fluids, especially for multiphase flows with strongly curved surfaces. It can be thought as a measure of the relative importance of the fluid's inertia compared to its surface tension. The quantity is useful in analyzing thin film flows and the formation of bubbles and droplets.

The Weber number is important for all single droplet simulations due to importance of surface tension in the impingement process. Other important dimensionless quantities include Reynolds number, Froude number, and Ohnesorge number. These quantities are determined as follows:

The Reynolds number is a dimensionless quantity that is used to help predict similar flow patterns in different fluid flow simulations. It is defined as the ratio of inertial forces to viscous forces.

$$
\operatorname{Re}=\frac{\rho v L}{\mu}
$$


The Froude number is a dimensionless number defined as the ratio of flow inertia to the external field (the latter in many applications simply due to gravity).

$$
\mathrm{Fr}=\frac{u_{0}}{\sqrt{g_{0 l_{o}}}}
$$

Ohnesorge $(\mathrm{Oh})$ is a dimensionless number that relates the viscous forces to inertial and surface tension forces.

$$
\mathrm{Oh}=\frac{\sqrt{W e}}{R e}=\frac{\mu}{\sqrt{\rho \sigma L}}
$$

The Prandtl number (Pr) is a dimensionless number defined as the ratio of momentum diffusivity to thermal diffusivity. It contains no length scale in its definition and is dependent only on the fluid and fluid state.

$$
\operatorname{Pr}=\frac{v}{\alpha}=\frac{c_{p} \mu}{k}
$$




\section{Volume of Fluid model (VOF)}

The VOF model (Hirt et al., 1981) is the most widely used model of gas-liquid or immiscible liquid-liquid flows in CFD. It is the interface tracking between two immiscible fluids. (This can be liquid-liquid interaction or gas-liquid interaction). The thesis discusses only liquid-gas interactions. Finding out whether a liquid is present in a cell is relatively easy. But, when the mesh is so fine, a great deal of computational power and time are required to find if the liquid is present in all the cells. So, a variable called liquid volume fraction is defined. The volume fraction is defined in such a way that it takes the value of 1 when the cell is filled with liquid, and it takes the value of 0 when the cell is completely filled with gas. If the variable varies between 0 and 1 , there must be a liquid-gas interface somewhere within the cell field.

In the VOF model, a single set of momentum equations is shared by the fluids and the volume fraction of both gas and liquid in each computational cell is tracked throughout the domain. Some applications of VOF model are free-surface flows, filling of a tank, sloshing, the motion of large bubbles in a liquid, the motion of liquid after a dam break, the prediction of a jet break-up (due to surface tension) and the steady state tracking of any liquid-gas interaction. 


\subsection{VOF model in OpenFOAM}

In the present thesis, the simulations are done in OpenFOAM-4.0.0, which is open source CFD software. It implements the VOF model. Taking into account the set of momentum equations and the variable for the volume fraction of the liquid, the VOF model can model the behavior and interaction of any number of immiscible fluids in a given domain. The number of variables for the volume fraction of the liquid increases as the number of fluids increase. For any given computational cell in the domain, the volume fractions of all the fluids sum up to unity. For any given fluid identified by the value of sub script " $n$," its volume fraction is denoted by " $\alpha_{n} "$ and satisfies the following:

$$
\begin{aligned}
& \alpha_{n}=1 \rightarrow \text { the cell is filled completely with } \mathrm{n}^{\text {th }} \text { fluid } \\
& \alpha_{n}=0 \rightarrow \text { the cell doesn't contain any } \mathrm{n}^{\text {th }} \text { fluid } \\
& 0 \leq \alpha_{n} \leq 1 \text { for any given computational cell }
\end{aligned}
$$

With the value of $\alpha_{n}$ denoting the fraction of cell occupied by fluid ' $n$ '.

The location of the interface, its properties, and shape can be solved. The location can be known by solving the continuity equation for each phase. The continuity equation of the $\mathrm{n}^{\text {th }}$ phase is as follows: 
$\frac{1}{\rho_{n}}\left[\frac{\partial\left(\alpha_{n} \rho_{n}\right)}{\partial t}+\nabla \cdot\left(\alpha_{n} \rho_{n} \overrightarrow{\left.v_{n}\right)}\right]=S_{\alpha n}+\sum_{m=1}^{r}\left(\dot{m}_{m n}-\dot{m}_{n m}\right)\right.$ (3.2)

The term ' $\rho_{n}$ ' indicates the density of the $\mathrm{n}^{\text {th }}$ fluid. The $S_{\alpha n}$ is the source term, which is set to zero since there is no mass production or loss in the $\mathrm{n}^{\text {th }}$ phase due to condensation or boiling in the present work. The $\dot{m}_{m n}, \dot{m}_{n m}$ terms are the rates of mass transfers from $m$ to $n$ and $n$ to $m$ respectively.

The sum of all volume fractions in a given domain cell is unity:

$$
\begin{aligned}
& \sum_{m=1}^{r} \alpha_{m}=1 \\
& r=\text { no. of fluids }
\end{aligned}
$$

There is a single momentum equation as well as $(r-1)$ continuity equations throughout the domain, which can be solved either implicitly or explicitly. There are many discretization methods available to discretize the equations in both implicit schemes as well as explicit scheme. A reconstruction scheme known as Geometric Reconstruction Scheme (Youngs,1982) is used for the present work. The main aim of this scheme is to calculate the location and shape of the interface 
exactly. Both implicit and explicit schemes treat all the cells equally. A piece-wise linear approach is used to represent the interface. A linear slope is assumed in each cell for the interface between the phases. This linear shape is then used to find the mass transfer to and from the cells.

Scalar properties in the transport equations for each cell are calculated using the volume fractions of the fluids present in the corresponding cell. The following equations give the volume fraction mean quantities throughout the cells:

$$
\begin{aligned}
& \rho=\sum_{n=1}^{r} \alpha_{n} \rho_{n} \\
& T=\sum_{n=1}^{r} \alpha_{n} T_{n} \\
& \mu=\sum_{n=1}^{r} \alpha_{n} \mu_{n}
\end{aligned}
$$

For velocity, a momentum equation is solved. The equation is coupled with the volume fraction through the density and viscosity terms.

$$
\frac{\partial(\rho \vec{v})}{\partial t}+\nabla \cdot(\rho \vec{v} \vec{v})=-\nabla p+\nabla \cdot\left[\mu\left(\nabla \vec{v}+\nabla \overrightarrow{v^{T}}\right)\right]+\rho \vec{g}+\vec{F}
$$


where $\vec{F}$ is the body force and $\vec{g}$ is the acceleration due to gravity.

The continuity equation is given by,

$$
\nabla \cdot \vec{v}=0
$$

Surface tension forces and viscous forces affect the equation because of the volume fraction term. It is calculated as the:

$$
\overrightarrow{F_{S}}=\sigma \kappa(x) \vec{n}
$$

where,

$$
\begin{aligned}
& \vec{n}=\frac{\Delta \alpha}{\Delta|\alpha|^{\prime}} \text { where } \vec{n} \text { is the unit vector normal to the surface. } \\
& \kappa(x)=\nabla \cdot \vec{n} \text { is the curvature of the surface. }
\end{aligned}
$$

The volume of fluid ' $n$ ' present in the cell is determined as follows:

$$
\underset{n}{F_{\text {vol }}}=\alpha_{n} V_{\text {cell }}
$$

where $V_{\text {cell }}$ is the volume of the cell.

Using the scalar transport equation, the volume fraction can be calculated by solving the follow equation: 


$$
\frac{\partial \alpha_{n}}{\partial t}+\nabla \cdot\left(\alpha_{n} \vec{v}\right)=0
$$

The solution of the energy equation is included in several solvers in OpenFOAM for compressible flow, combustion, heat transfer, multiphase flow and particle tracking. The following is the energy equation:

$$
\rho \frac{D e}{D t}+\rho \frac{D K}{D t}=-\nabla \cdot q+\nabla \cdot(\sigma \cdot U)+\rho g \cdot U
$$

where $\mathrm{U}$ is the velocity, $\mathrm{K}$ is the specific Kinetic Energy, $\sigma$ is the mechanical stress tensor and $\boldsymbol{g}$ is the body force acceleration. 


\section{Problem Description}

This chapter introduces the problem that has been studied in this thesis. As explained earlier, simulating sprays is highly time-consuming and needs high computational power. A Monte-Carlo (MC) model of spray has been developed by Krietzer (2010) and by Hussain et al. (2014) to simulate sprays. The MC model reduces the cost and increases the efficiency. Keeping aside the complexity of the spray, the behavior of a single droplet on the residual layer has been simulated as a simplified building block of the full spray process. In the present study, we need to find the cavity life-time, the sub-cavity liquid thickness which leads to subcavity liquid volume, and temperature profile as functions of time.

Depending on the values of droplet impact Weber number, Reynolds number and Froude number and the non-dimensional height of the liquid film (h/d), five types of single droplet simulations have been made for temperature profile, cavity lifetime, sub-cavity film thickness and the computed sub-cavity film volume. Weber number in this study is varied from 135 to 993, Reynold's number is varied from 1180 to 3550 , and Froude number is varied from 94 to 510 . Values of the initial two numbers have the dominant influence on the time histories of the sub-cavity film thickness and volume. 
The ranges of We and Re are taken as such because we are comparing the present results with the experimental results (Hillen, 2013), which are done between the same ranges. They are done between the same ranges because of the apparatus used for the experiments. The pressure in the apparatus are able to produce droplets with a minimum Weber number of 135 and maximum weber number around 1000.

The present simulations have been categorized into five cases based on the increasing value of Weber numbers (Case 1 has the lowest Weber number, and the Case 5 has the largest Weber number). Each of these five cases has been subdivided again into three cases based on the values of a non-dimensional height of the liquid layer (h/d): Case a has $h / d$ equal to 1.0 , Case $b$ has $h / d$ equal to 0.5 and Case $\mathrm{c}$ has $\mathrm{h} / \mathrm{d}$ equal to 0.2 . Based on these values, the droplet impact velocity, the diameter of the drop and the film thickness are calculated. From all of these single droplet simulations, we hope to be able to predict the amount of heat transferred from the heated surface by the full spray. All these cases have been simulated by taking the energy equation into consideration. 


$\begin{array}{llll}\text { Case } & \begin{array}{l}\text { Initial Film Thickness } \\ (\mu \mathrm{m})\end{array} & \begin{array}{l}\text { Diameter of the } \\ \text { droplet }(\mathrm{mm})\end{array} & \begin{array}{l}\text { Velocity of } \\ \text { impingement }(\mathrm{m} / \mathrm{s})\end{array} \\ \text { Case 1 a } & 3020 & 3.03 & 1.73 \\ \text { Case 1 b } & 1530 & 3.06 & 1.73 \\ \text { Case 1 c } & 724 & 3.03 & 1.67 \\ \text { Case 2 a } & 3500 & 3.47 & 2.72 \\ \text { Case 2 b } & 1760 & 3.47 & 2.73 \\ \text { Case 2 c } & 714 & 3.48 & 3.72 \\ \text { Case 3 a } & 3010 & 3.09 & 3.55 \\ \text { Case 3 b } & 1550 & 3.08 & 3.55 \\ \text { Case 3 c } & 606 & 3.11 & 3.66 \\ \text { Case 4 a } & 3120 & 3.49 & 3.69 \\ \text { Case 4 b } & 1730 & 3.47 & 3.72 \\ \text { Case 4 c } & 695 & 3.46 & 3.72 \\ \text { Case 5 a } & 3160 & 3.52 & 4.20 \\ \text { Case 5 b } & 1750 & 3.52 & 4.16 \\ \text { Case 5 c } & 707 & 4.18\end{array}$

Table 4.1 List of all cases with the values of the parameters 


\section{Implementation of OpenFOAM Codes}

The 3-D simulations, with the energy equations, are done in an open source software called OpenFOAM-4.0.0. As the simulation is 3D, there are a huge number of cells which results in longer execution times. So the OpenFOAM simulations are done with the help of High-Performance Computing Center at the West Virginia University with eight processors.

As already discussed in Chapter 3, the VOF model is used for the simulations. Two phases are used: liquid and air. For the purpose of tracking the interface, Euler scheme is used, which is also briefly explained in Chapter 3. The maximum Courant number at the free surface is specified as 0.25 . The density, dynamic viscosity of liquid and air are taken as $997 \mathrm{~kg} / \mathrm{m}^{3}, 1.2041 \mathrm{~kg} / \mathrm{m}^{3}, 0.000998$ $\mathrm{kg} / \mathrm{m}-\mathrm{s}, 1.8 \mathrm{e}-05 \mathrm{~kg} / \mathrm{m}$-s respectively. The reference temperature and surface tension are taken to be $288 \mathrm{k}$ and $0.0723 \mathrm{~N} / \mathrm{m}$ respectively. To increase the wetting of the surface, the contact angle between the droplet and the liquid film is set to be 2 degrees. All the side and top surfaces of the domain are pressure outlet boundary conditions, whereas the bottom surface is the wall. The domain size is $36 * 18 * 36 \mathrm{~mm}(36 \mathrm{~mm}$ in $\mathrm{x}$ and $\mathrm{z}$ directions and $18 \mathrm{~mm}$ in vertical $\mathrm{y}$ direction) The mesh used in OpenFOAM was set to a geometric progression in the vertical direction due to restrictions on computational power and to maintain better resolution in the plane that contains the sub-cavity liquid film thickness. The lowest cell edge length was set to $d / 100$ at the bottom wall, increasing to $d / 5$ at the top wall.

compressiblelnterFoam is the solver that is used to track the interface between the droplet and the liquid film. As explained in Chapter 3, the VOF model 
is used to track the interface. This scheme concentrates only on the interface. Fig 5.1 shows the boundary conditions and domain for the simulations.

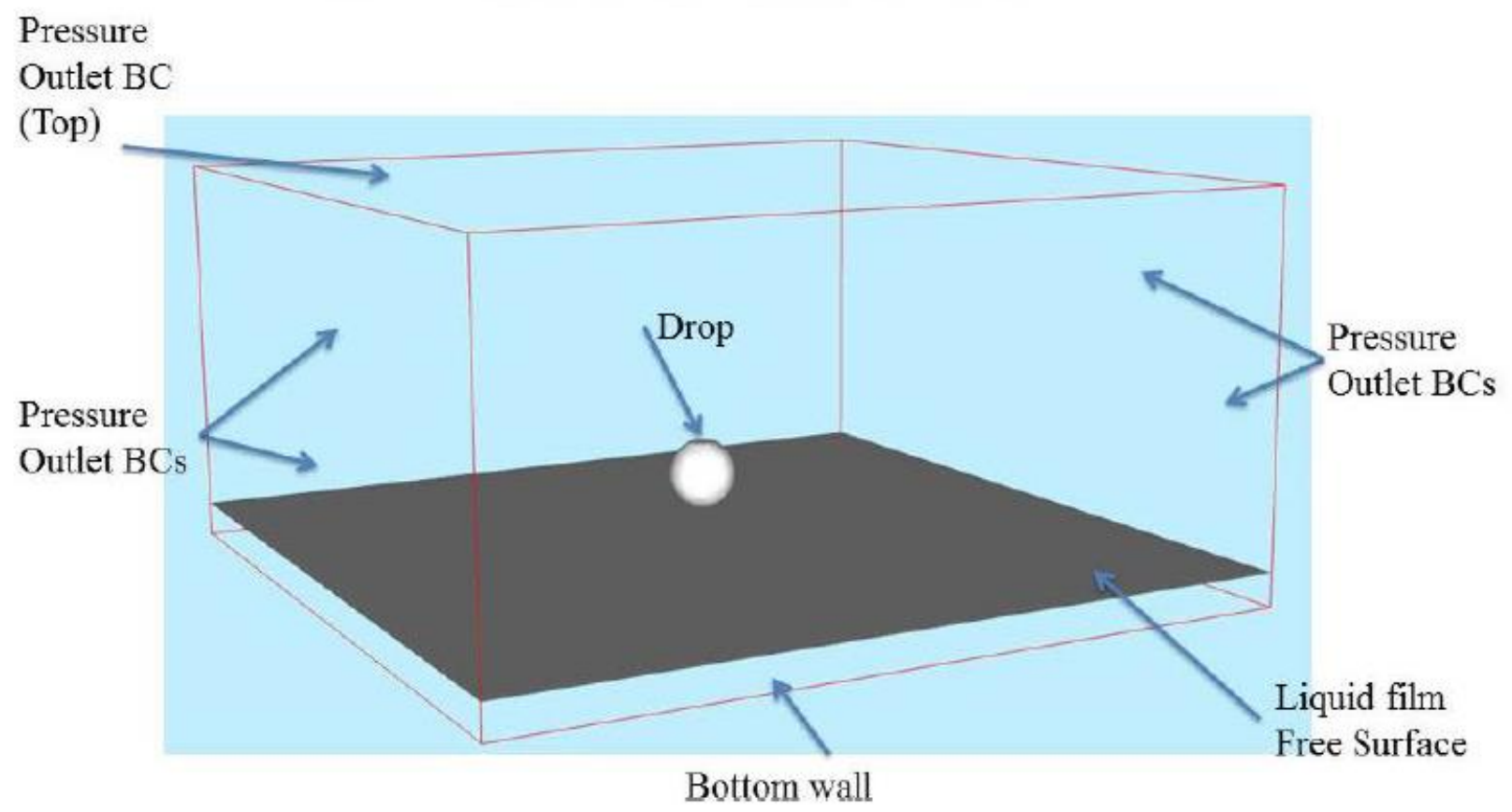

Fig. 5.1 Boundary conditions for the simulation

The basic directory structure for any OpenFOAM case are the time directories, a constant directory and a system directory. The time directories are present in the folder 0 . It contains individual files of data for particular fields. The data can be initial values or boundary conditions. It defines the state of flow initially. The constant directory contains description about mesh and physical properties required for the application. The contents of the constant folder remain the same throughout the simulation. The system directory contains the parameters like start and end times, time steps, the discretization schemes, equation solvers, 
tolerances and other algorithm controls, which are associated with the procedure itself.

\subsection{Domain \& Mesh}

The file, which contains the information about the domain, its vertices, type of mesh, is the 'blockMeshDict' in the system folder. The contents are present in the Fig. 5.2 below. The value of 'convertToMeters' is specified to be 1, which indicates that all the values in this file are in meters. The vertices of the domain are specified under the 'vertices' section. The first set of parenthesis in the block section specifies the numbering of the vertices, the first vertex being zero. The word 'hex' specifies that hexahedral cell is created. The second set of parenthesis specifies the number of mesh points in $x, y$ and $z$-axes respectively. The 'simpleGrading' gives information about how the mesh points are places. They are placed evenly in the $\mathrm{x}$ and $\mathrm{z}$-direction but are expanded in the $\mathrm{y}$-direction in a geometric progression. The value represents the ratio of lengths of the last cell to that of the first cell. In the 'boundary' section, the type of boundary associated with the faces containing respective vertices is mentioned. Fig.5.2 shows the required information to create the mesh.













Fig.5.2 blockMeshDict file

\subsection{Boundary Conditions}

The boundary conditions corresponding to the simulation is present in the ' $O$ '

folder. It has files of alpha.water, $p, p_{-} r g h, T, U$ which specifies the values of the volume fraction of water, pressure, hydrostatic pressure, temperature and Velocities respectively. They are placed in the ' 0 ' folder because their values are specified at time $=0$. Fig.5.3 below shows the file structure of alpha.water .






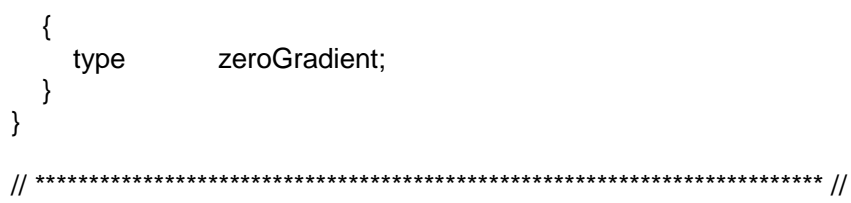

Fig. 5.3 alpha.water file

The values under the bottom section show that the liquid is in contact with the bottom wall with a constant contact angle of $2^{\circ}$. The front, back, and sidewalls are specified with a zeroGradient, which means there is no entry or exit of the liquid. The values of 'atmosphere' sections state that values are subject to change later. The internalField is stated to be uniform 0 , which tells us the value of alpha.water is zero in rest of the domain if not mentioned.

\subsection{Properties}

The contents of the constant folder specify the values of properties. It has files of g, thermoPhysicalProperties, thermoPhysicalProperties.air, thermoPhysicalProperties.water, turbulenceProperties. These files are placed in the constant folder because these values remain constant throughout the simulation. These files specify the values of acceleration due to gravity, density, specific heat capacity, kinematic viscosity, surface tension and all other thermal and physical properties in SI units unless specified.

\subsection{Control Dictionary}

The next step after specifying the boundary conditions, initial conditions and the properties is to specify the type of application used, time step and data save 
intervals for the simulation. All this information is present in the controlDict file in the system directory. Its file structure is shown in the Fig. 5.4 below. The type of application that is used for this simulation is compressiblelnterFoam. This is used for two immiscible, incompressible fluids within energy equation included. The runTimeModifiable and adjustbleTimeStep are specified to be yes, which means the run time and time step are subject to changes during the simulation, keeping the constraint of maximum Courant number, set at maxCo. The time step is also constrained by maxDeltaT, which indicates the maximum size of the time step. The end time is specified to be ten times the total flow-through time of each simulation.




writePrecision 6;

writeCompression compressed;

timeFormat general;

timePrecision 6;

runTimeModifiable yes;

adjustTimeStep yes;

$\max C o \quad 0.5$;

maxDeltaT $1 ;$

maxAlphaCo 0.5 ;

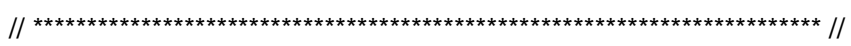

Fig. 5.4 controlDict file

\subsection{Parallel Computation.}

The current mesh generated contains around 1 million cells per each time step for each case which requires high performance computation. At West Virginia University (WVU), a Super Computing System, called Mountaineer, is funded in part by the National Science Foundation EPSCoR Research Infrastructure Cooperative agreement \#1003907, the state of West Virginia (WVUEPSCoR via the Higher Education Policy Commission) and WVU was available. Some of the simulations are done in the Mountaineer HPC and the remaining are done in Google Cloud. Eight processors with a total of $48 \mathrm{~GB}$ of RAM has been used in the Mountaineer HPC and eight processors with a total 52GB RAM has been used in the Google Cloud to compute the 3D simulations. The information about parallel processing are present in the decomposeParDict file in the system directory, Fig.5.5. The simple is the type of method used to divide the case into 8 subdomains. The value of $\mathrm{n}$ in the simpleCoeffs is specified to be $\left(\begin{array}{ll}2 & 2\end{array}\right)$ which means the domain is divided into two subdomains in each of $x, y$ and $z$ directions. The term delta specifies the maximum distance between the divided subdomains 


\section{that can be applied. The simple method divides the domain equally into 8}

\section{subdomains.}



Fig.5.5 decomposeParDict file 


\subsection{Numerical Schemes}

There are a large number of numerical schemes, available in OpenFOAM for the user to calculate all the derivatives in the equations approximately. These schemes are specified in the fvschemes file in the system directory; see Fig.5.6. Different schemes can be used for different type of derivatives present in the equation.

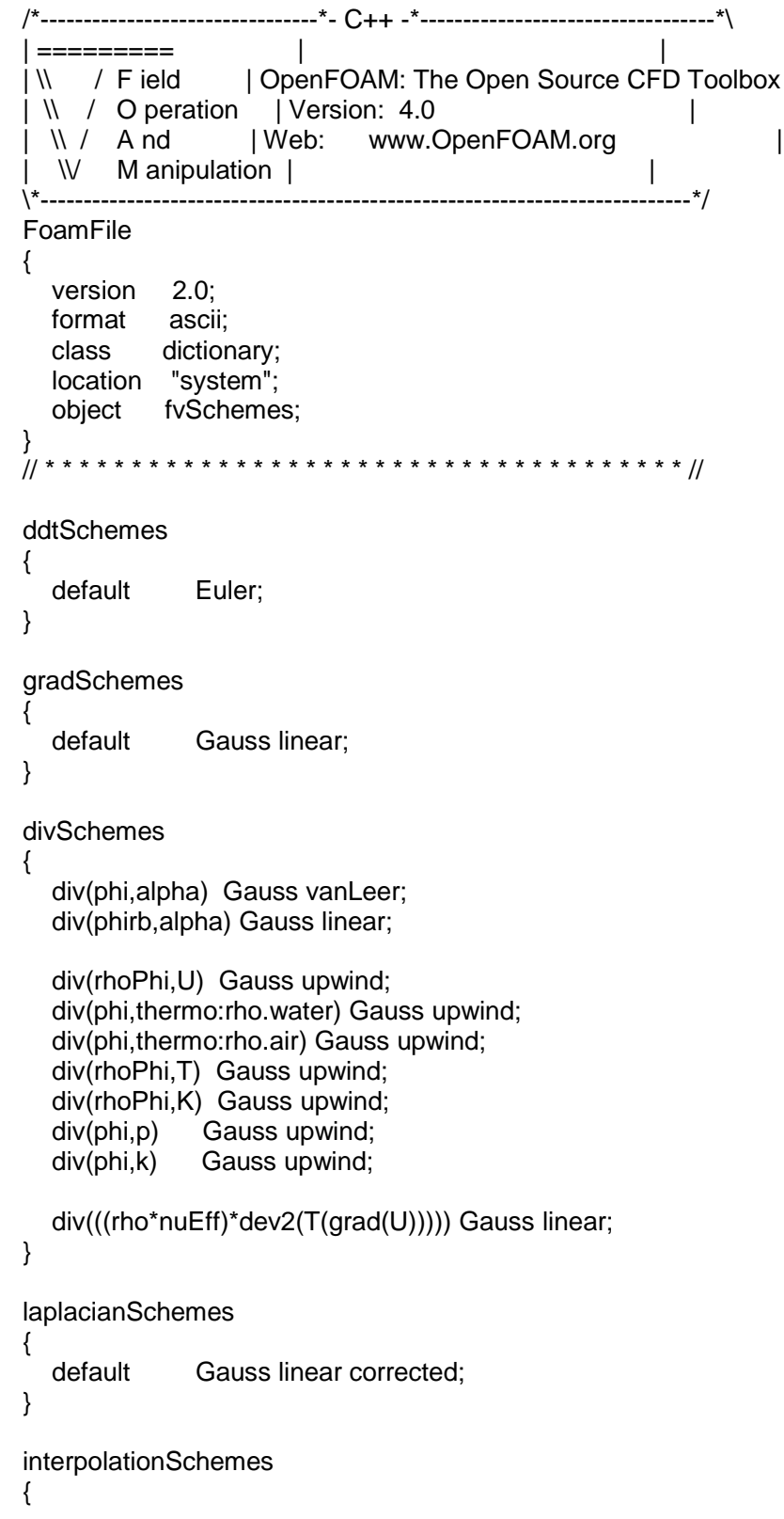




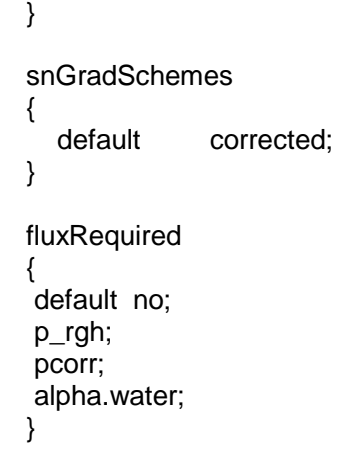

Fig.5.6 fvschemes file

This scheme uses the values on the faces of the cell and adds them up. These values are interpolated from the neighboring cells. Linear interpolation is used in most of the cases. All the time derivatives are evaluated using Euler scheme. It is implicit and first order. All the gradient terms are calculated approximately using the Gaussian scheme and linear interpolation is done by default. All the divergence terms in the equations are done by using the schemes mentioned in the divSchemes section. The Laplacian terms in the Navier-Stokes Equation are solved by using the schemes mentioned in the laplacianschemes section. The terms in under the interpolationSchemes defines how the interpolations are made. snGradSchemes section represents how surface normal gradient terms are calculated. corrected scheme is used because it is conservative, unbounded and second order in nature. The values which require the generation of flux, which are alpha, corrected pressure, pressure without the buoyant pressure, are mentioned in fluxRequired section.

\subsection{Setting the Fields}

The terms that are used to specify the values of the liquid film thickness, center of the drop, its radius and velocities are all specified in setFieldsDict file in the system directory, shown in Fig.5.7 


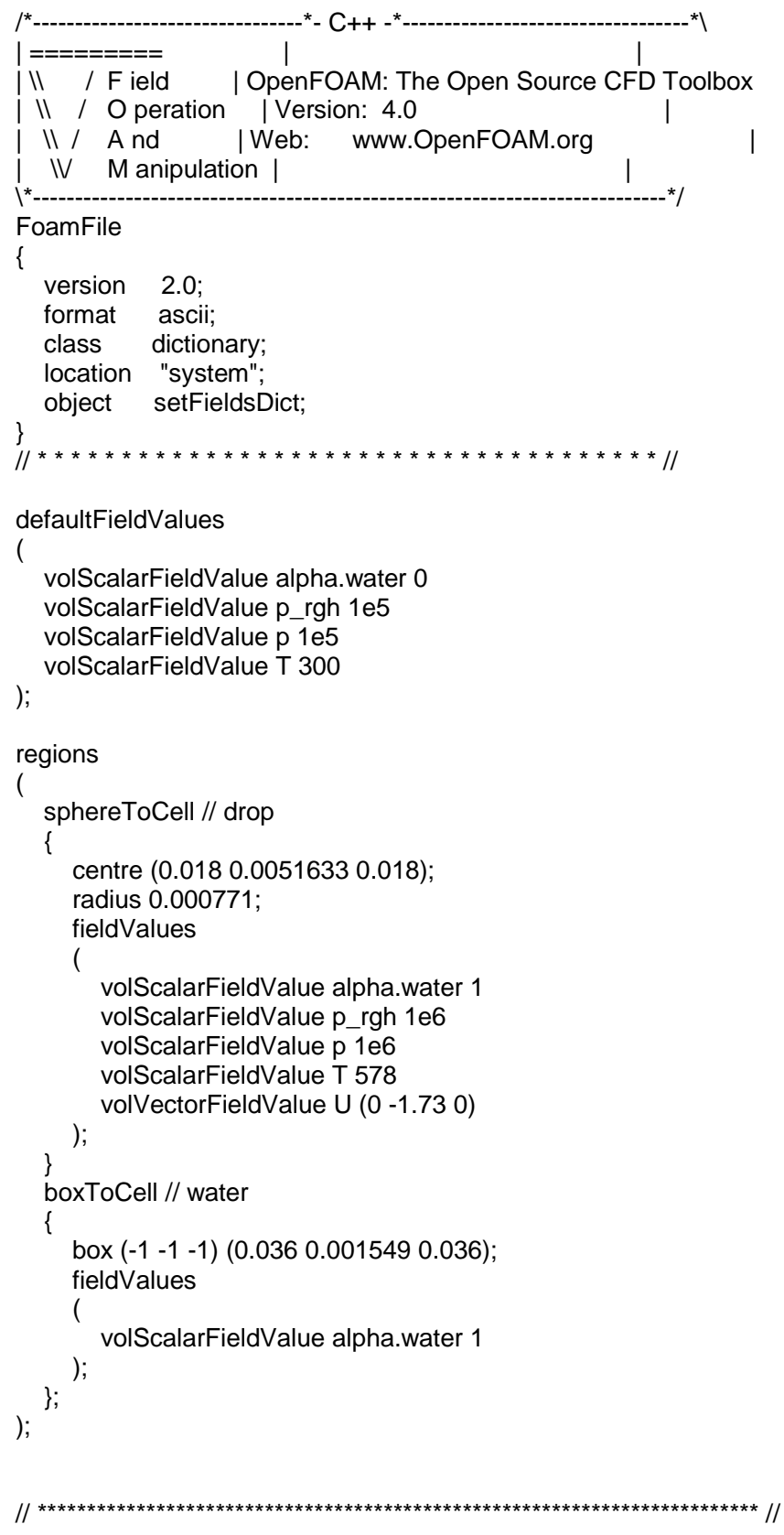

Fig. 5.7 setFields file

The defaultFieldValues specifies the default values in the domain when not

specified. The sphereToCell section in the regions sections specifies the parameters of the droplet and boxToCell represents the parameters of the liquid

film. 


\subsection{Solvers}

The solvers needed to solve the required variables, along with the tolerances, algorithms and residuals are mentioned in the fvSolution file in the system directory: see Fig.5.8. The beginning of every variable, there is a solver keyword which specifies the method used by the OpenFOAM to solve the matrices obtained from the equation. compressibleInterFoam solves the equation to find out alpha.water, pcorr, p_rgh,p_rghFinal and U. For each of these variables, a solver, preconditioner, tolerance, relative tolerance and maximum number of Iterations and smoothers are mentioned in the respective sections. A preconditioner is used to reduce the complexity of the problem to a form, which can be solved by simple numerical methods.






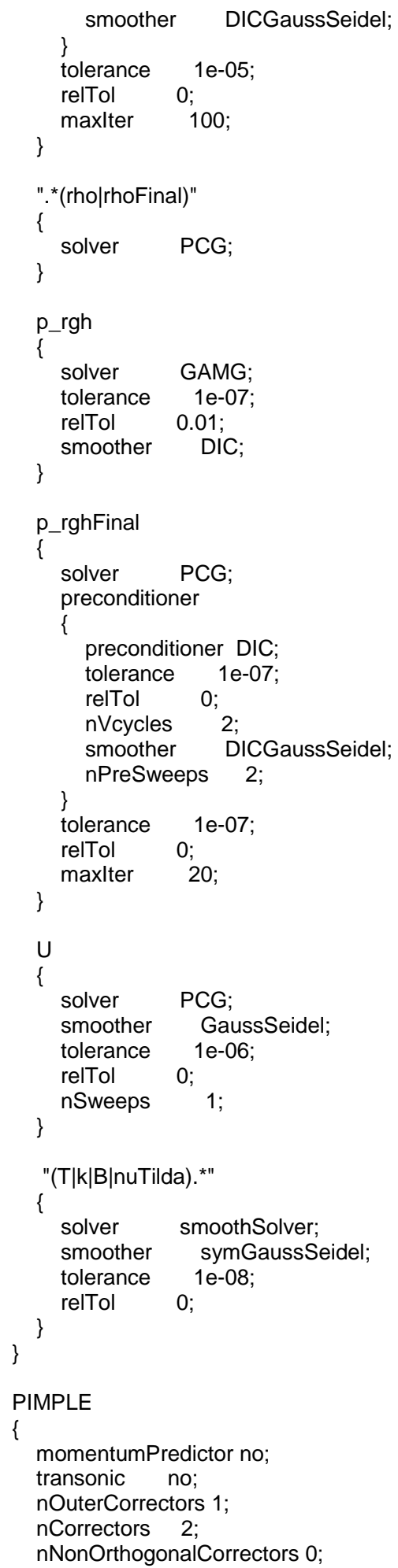

Fig.5.8 fvSolution file 
All the numerical methods are iterative and the iterations will be stopped when it meets all criterion mentioned in the tolerance, relTol and maxlter are met. Iterations stops when the tolerance exceeds the specified tolerances, iterations exceed the value of maxiter or when the ratio of residuals fall below relTol. The lower the value of relTol, the higher the accuracy.

The PIMPLE algorithm is a combination of PISO and SIMPLE algorithms. The number of corrections to be made are mentioned in the $n$ Correctors. Since all the simulations are done along the coordinate axes, no orthogonal corrections are made. The value of alpha is calculated as many times as specified by the user for the keyword nAlphaSubCycles. To improve the quality of alpha, it is iterated through fixed point iteration by the number specified for the keyword nAlphaCorr. The cAlpha specifies the interface compression. 


\section{Post Processing and Analysis}

Next task after simulations are done is to analyze the data to compute the required parameters from the cavity formation. For analysis, the simulated data is to be exported to a software that can analyze and visualize the simulated data. Paraview is used to visualize the simulated data, and MATLAB is used to get the required parameters from the simulations. Paraview is an open source software which can be downloaded along with OpenFOAM.

In this thesis, the simulated data is visualized and the data of volume fraction, temperature, pressure and velocities at each mesh point in the domain is predicted in Excel files. This data is exported into excel and analyzed in MATLAB to calculate the required parameters. To find out the film thickness and the subcavity volume at each time step, the crown and cavity formation should be sorted out. Interface location is defined as where the volume fraction of water is 0.5 . Since, the cavity is formed along the interface, it is enough if we analyze this data where alpha.water is 0.5 .

Since the simulations are 3D, the whole domain is divides exactly into two halves, cutting plane along the XY plane passing through the center of the domain using the 'slice' option in Paraview.

\subsection{Centerline Film Thickness}

The centerline film thickness is the thickness of the liquid film that lies under the cavity. So, the interface is created where the value of volume of fraction is 0.5 . The value of centerline film thickness is calculated in MATLAB. Since the domain 
in divided into two halves, the droplet and the cavity are also divided into two parts. The problem is now converted into a $2 \mathrm{D}$ problem in the plane containing the center of the droplet. The thickness can be calculated by finding the point along interface, which is at the lowest height from the bottom wall. Eventually, the $y$-coordinate of the point gives the thickness of the film below the cavity. This point also a fixed $x$ and $z$ coordinates since this point lies on the line parallel to $y$ axis and passing through the center of the domain. This is the algorithm used to find the film thickness, and a MATLAB code has been written to perform the calculations.

\subsection{Sub-cavity Volume}

Time dependent sub-cavity volumes were obtained by numerically integrating the cavity film thickness data at fixed time versus the cavity radius. Since the subcavity volume is the quantity of interest, the measured volumes when droplet is initially forming the cavity, and after the cavity has filled back in haven't been calculated. Sub-cavity volumes for specific times were computed using the trapezoidal rule, in MATLAB, using

$$
V_{o l}=2 \pi \int_{0}^{R_{c_{i}}}(R * h) d R
$$

\subsection{Average Heat Flux}

The average heat flux is calculated near the bottom wall beneath the cavity. The temperature of the bottom wall is fixed to be $372 \mathrm{~K}$ (just below the boiling point of water). A code is written in MATLAB to find the temperature profile in a plane just 
one mesh point above the bottom wall. So the temperature difference can be found which leads to the heat flux.

$$
\mathrm{Q}=-\mathrm{k} \mathrm{A} \frac{T_{w a l l}-T_{\Delta y}}{\Delta y}
$$

The average heat flux is calculated for the liquid which is under the cavity and also outside the cavity. 


\section{Results}

\subsection{Overview}

This chapter presents and discusses the results of the present thesis. When a drop impacts the liquid surface, due to the momentum of the droplet, the static liquid layer is pushed down creating a cavity. The layer of liquid below the cavity is termed as the "sub-cavity liquid layer" in the present work. Also, due to the temperature differences, on a whole energy differences, there will be energy exchange between the static liquid and the droplet. The thickness of this thin layer is of interest, as a region of high transient heat flux in spray cooling applications (Kuhlman et al., 2007). After reaching the impact surface, the formation of the cavity leads to the formation of crown at sufficiently high Weber numbers. Crown is formed as a result of liquid volume displaced from where the cavity is formed. The cavity radius increases and the cavity starts to spread until all the kinetic energy from the drop impact has been converted into the potential energy, the increased surface energy, and viscous losses in the crown and the static liquid. This phase is the cavity formation phase. The crown that is formed depends on the values of the Weber number, Froude number and the static layer thickness. Crown formation can't be observed at relatively low Weber numbers (Medam, 2015). The height of the crown, in the case of high Weber number cases is largely determined by the values of surface tension and droplet impinging velocity. The thickness of the film and the value of the maximum radius of the cavity determine the value of sub-cavity volume, the determination of which is one of the main aims of the thesis.

When the droplet's kinetic energy is completely dissipated into the surrounding liquid, the further formation of cavity will be stopped. The surrounding 
liquid now starts to exert pressure on the edges of the cavity. And also, the surface tension and gravity pulls the crown downwards. Therefore the crown collapses and the cavity shape changes from a bowl to an inverted bell. All the liquid in the crown starts accelerating towards the center of the cavity and a central Worthington jet is often formed.

The temperature of the droplet is assumed to be $300 \mathrm{~K}$ and the temperature of the bottom wall is set to $372 \mathrm{~K}$. Therefore, the liquid layer heats up and there is a temperature difference between the droplet and the liquid film which leads to a heat transfer. After some amount of time, the liquid layer attains a fixed saturated temperature, which is less than $372 \mathrm{~K}$. More of these liquid droplet impacts reduce the temperature even faster. A second main aim of this thesis is to find what the heat flux is.

\subsection{OpenFOAM Visualization}

In this section, some of the results which are taken from the visualization of the CFD simulations can be seen. These results are taken from Paraview, the visualization software that is used.

As mentioned earlier, the simulation starts from time $t=0$ and ends at time $t=60 \mathrm{~ms}$. These set of figures below shows various stages of the simulation. These set of screenshots are taken for the case $3 \mathrm{~b}$, which has $\mathrm{We}=621, \mathrm{Re}=2643$, $h / d=0.5$ 


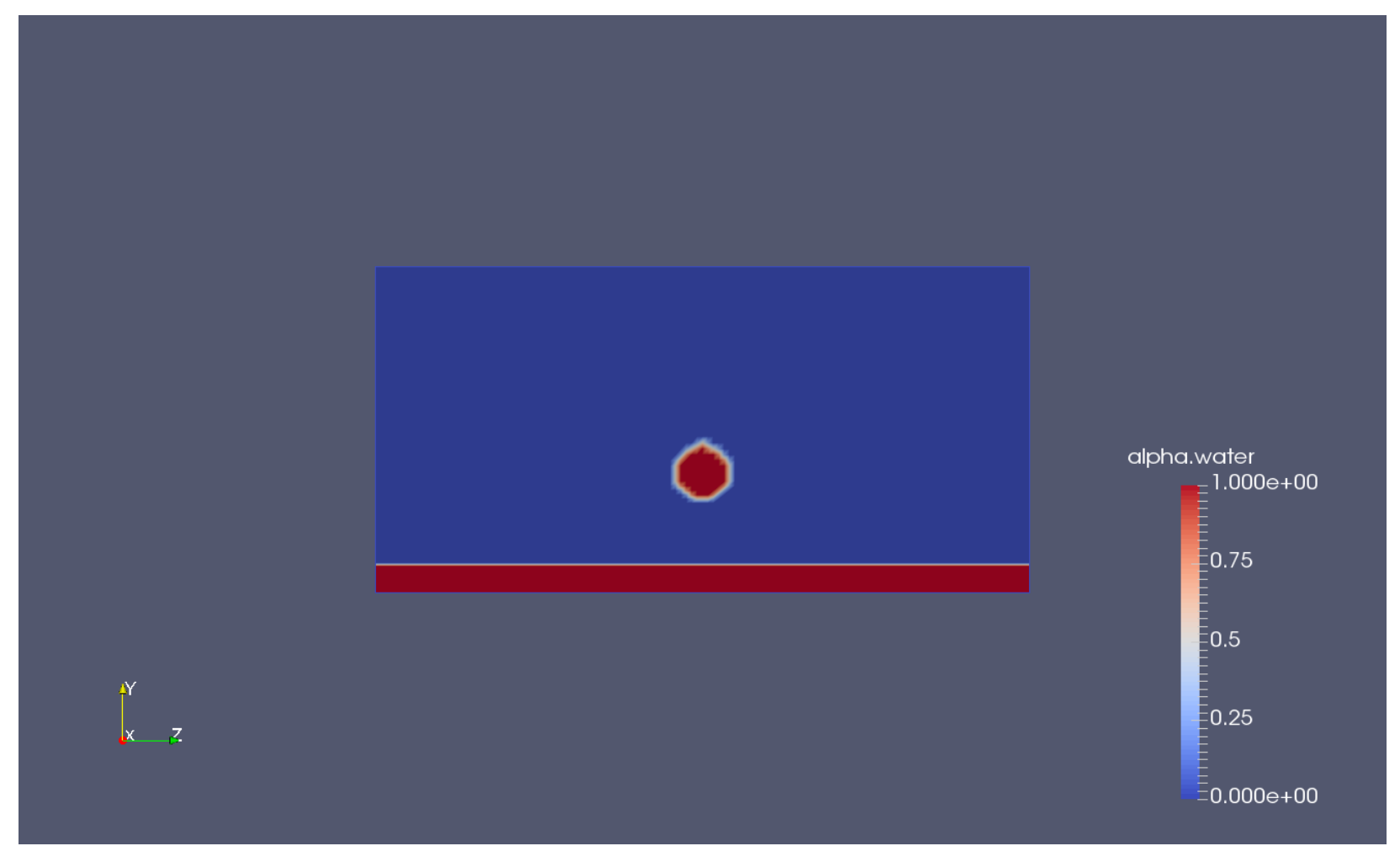

Fig. 7.1 time $=0$ for case $3 b(W e=621, R e=2643, h / d=0.5)$

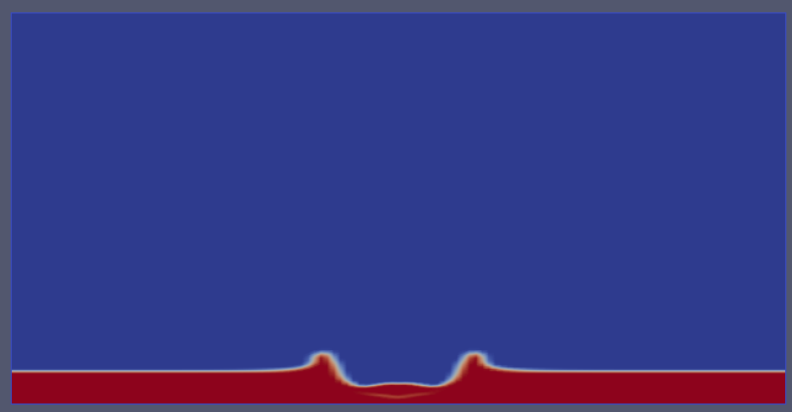

alpha.water

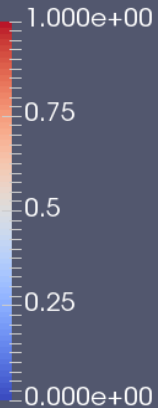

Fig. 7.2 time $=2.25 \mathrm{~ms}$ for case $3 b$ (We=621, $R e=2643, h / d=0.5$ 




alpha.water

Fig. 7.4 time $=15 \mathrm{~ms}$ for case $3 b(W e=621, R e=2643, h / d=0.5)$



alpha.water

$=1.000 e+00$

0.75

Fig. 7.5 time $=17 \mathrm{~ms}$ for case $3 b(W e=621, R e=2643, h / d=0.5)$ 


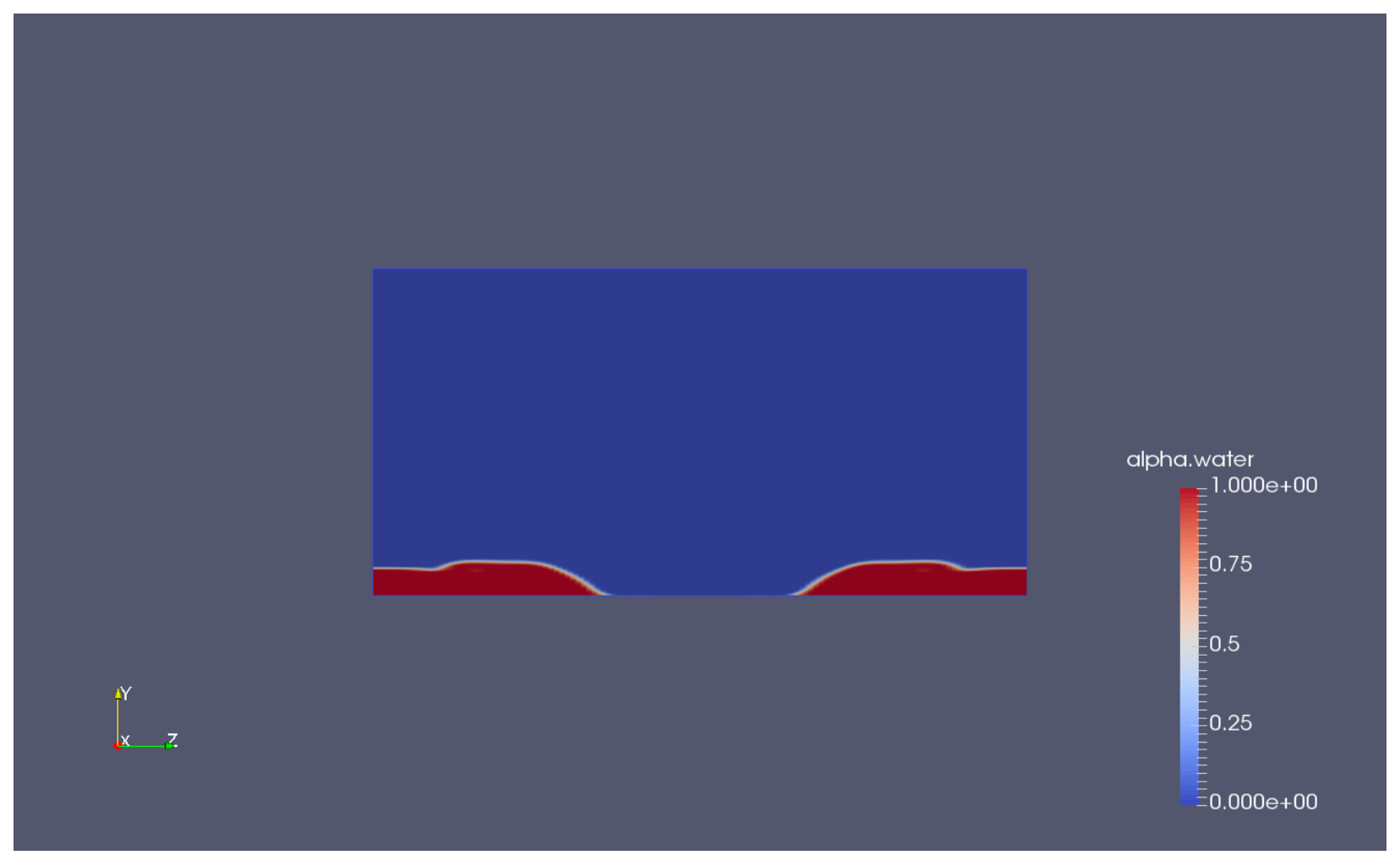

Fig. 7.6 time $=24 \mathrm{~ms}$ for case $3 b(W e=621, R e=2643, h / d=0.5)$ 


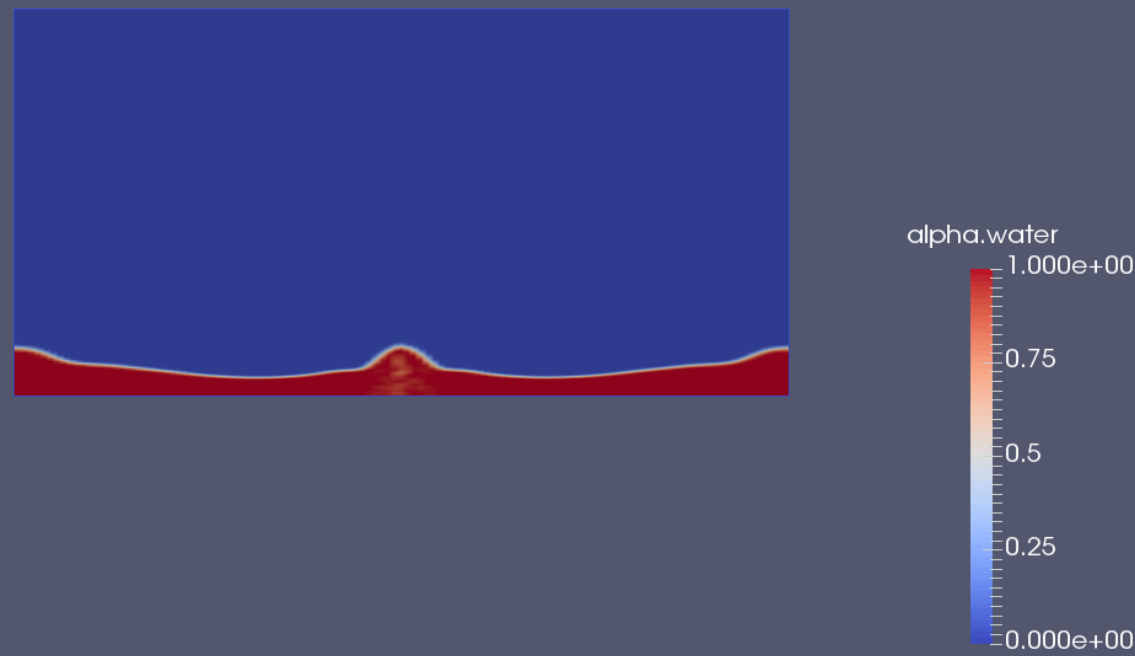

Fig. 7.7 time $=45$ ms for case $3 b(W e=621, R e=2643, h / d=0.5)$

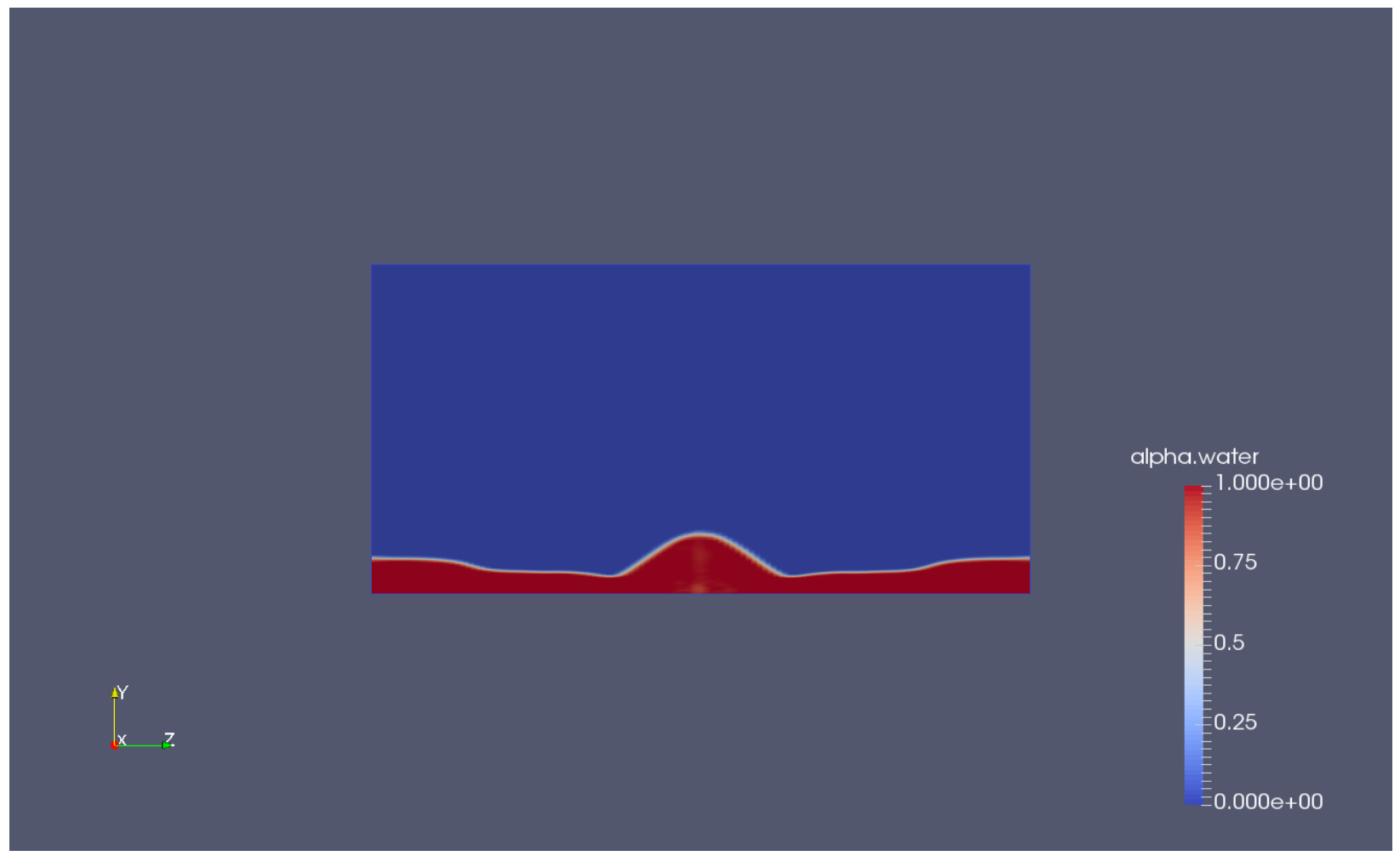

Fig. 7.8 time $=60 \mathrm{~ms}$ for case $3 b(W e=621, R e=2643, h / d=0.5)$ 
In Fig. 7.1, the initial position of the droplet and the liquid layer setup can be seen at time $t=0$. In Fig. 7.2, the impact of droplet onto the surface of the liquid layer can be seen at time $t=1.5 \mathrm{~ms}$. Fig. 7.3 and Fig 7.4 shows the formation of the crown at times $t=2.25 \mathrm{~ms}$ and $t=15 \mathrm{~ms}$ respectively. Fig. 7.5 shows the point where the cavity almost reached its maximum radius and the crown is about to collapse at time $t=17 \mathrm{~ms}$. Fig. 7.6 shows collapse of the crown at time $t=24 \mathrm{~ms}$.

Fig. 7.7 shows the complete collapse of the crown and the Worthington jet is about form at time $t=45 \mathrm{~ms}$. In the Fig. 7.8, the simulation has ended at time $t=60 \mathrm{~ms}$ and Worthington jet is clearly seen.

The figures below show the contour plots of the liquid fraction alpha (Fig.7.9) and temperature profiles (Fig.7.10) in the plane that is one mesh away from the bottom wall i.e., $y=35.183 \mu \mathrm{m}$. These contour plots are taken at time $t=20 \mathrm{~ms}$ for the case $3 \mathrm{~b}$.



Fig. 7.9 Contour plot of alpha for case $3 \mathrm{~b}$ at time $t=20 \mathrm{~ms}$ and in the plane $y=35.183 \mu \mathrm{m}$ 




Fig 7.10 Contour plot of temperature for case $3 \mathrm{~b}$ at time $t=20 \mathrm{~ms}$ and in the plane $y=35.183 \mu \mathrm{m}$ The clear formation of cavity can be seen in Fig. 7.9. In the Fig. 7.10, it could also be seen that the temperature just outside the cavity is lower whereas the temperature in the sub-cavity is higher which explains the need for spray cooling. A spray contains many droplets like these which cools the component much quicker than here, by heating the sub-cavity liquid faster than the static liquid layer.

\subsection{Comparison of Centerline Film thickness}

After presenting what the simulation does visually in the previous section, the next thing was to compare these CFD results with the experiments. The centerline film thickness is the height of the liquid layer above the bottom wall at the center of the cavity. This gives the information about the life time of the cavity and the formation of the Worthington jet. This section will discuss the centerline thickness histories 
of both the present CFD simulations and experiments Hillen (2013) and comparing them. The normalized centerline film thickness is calculated by:

$$
h^{*}=h / d
$$

The non-dimensional time is calculated as follows:

$$
t^{*}=t \frac{V e l}{d}
$$

Fig. 7.11 shows the dimensionless centerline film thickness profiles for 3D OpenFOAM case and experimental plots for the case $1 \mathrm{a}$. The fluent results and the OpenFOAM without the energy equation are from Medam (2015). It is observed that the thickness value falls, obviously, during the cavity formation and later increases as the crown collapses. It overlaps with those of the experiments during the initial stages. The OpenFOAM solution overshoots the cavity lifetime. It is speculated that due to the variation in surface tension of the liquid used in experiments, because of the laboratory environment, the cavity life time is shorter and the maximum height reached by the Worthington jet is also less because the surface tension is maintained constant throughout the simulation (Medam, 2015). 


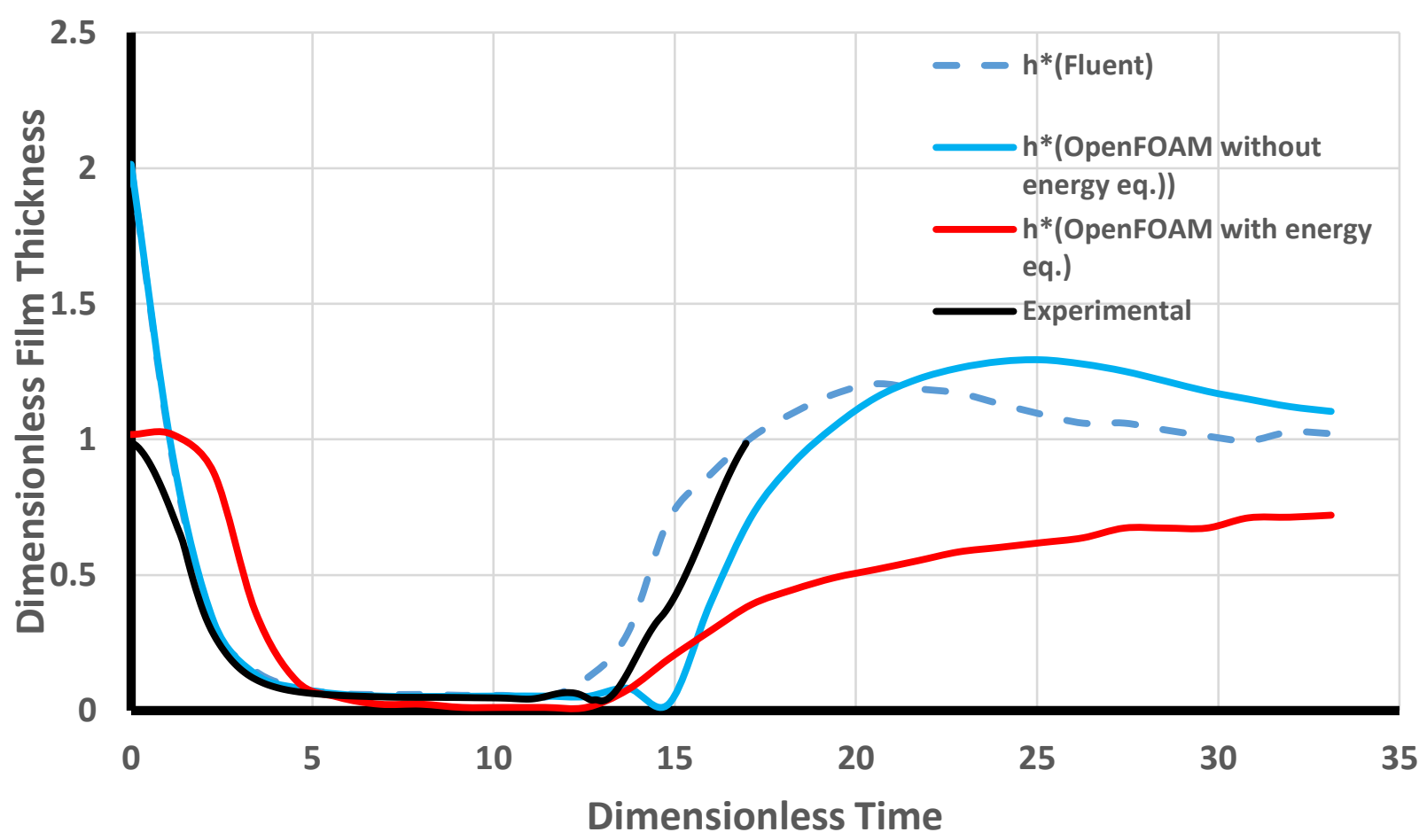

Fig 7.11 Centerline Film thickness for case $1 \mathrm{a}(\mathrm{We}=145, \mathrm{Re}=1189, \mathrm{~h} / \mathrm{d}=1.0)$

Centerline film thickness values of case $1 \mathrm{c}$ are shown in the Fig.7.12. As seen earlier, the values of CFD and experiments have coincided during the initial stages. Later, however the thickness values in the simulation are higher when compared to the experimental values. 




Fig 7.12 Centerline Film thickness for case $1 c(W e=135, R e=1176, h / d=0.2)$

Fig. 7.13 shows the variation of centerline film thickness with time for the case $3 \mathrm{~b}$. In this case, we observe a centerline film thickness makes an angle slightly greater than $90^{\circ}$. This steep profile is due to the increase in the droplet Reynolds' number. The velocity for this case is much higher than in the previous cases. So the Worthington jet is formed much earlier than in the previous cases. We also notice that the value of centerline film thickness is less when the cavity has been fully established. There are no cavity waves formed in the CFD simulations. 




Fig 7.13 Centerline Film thickness for case $3 b(W e=621, R e=2643, h / d=0.2$ )

Fig.7.14 shows the centerline film thickness for the case 5 a. Due to the higher impinging velocity, the cavity takes more time for its complete establishment. The simulation shows the formation of a smaller Worthington jet whereas a higher Worthington jet is formed in the experiments. 


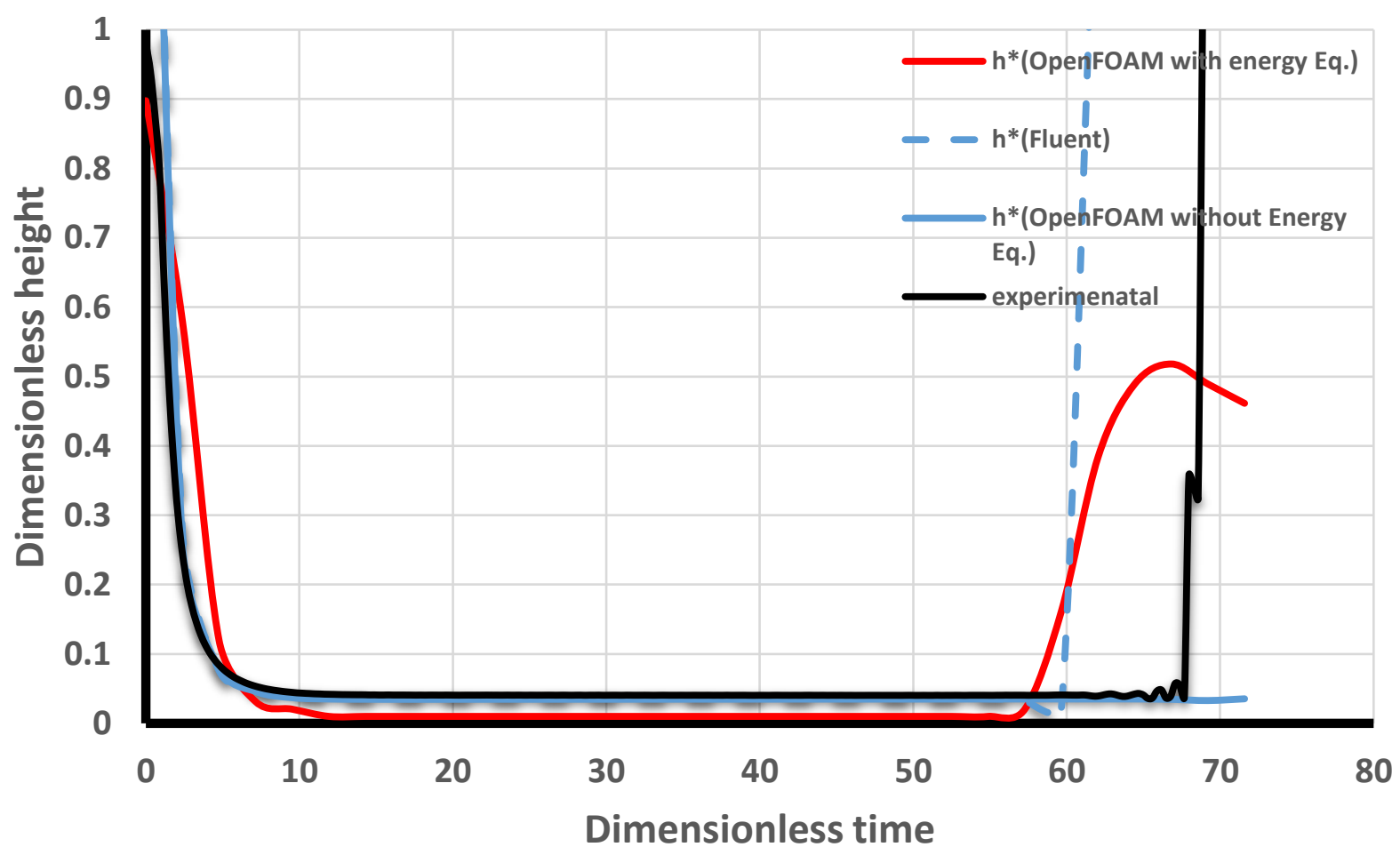

Fig 7.14 Centerline Film thickness for case 5 a $(W e=993, R e=3574, h / d=0.9)$

The Fig.7.15 below shows the time histories of centerline film thickness values of case $5 \mathrm{c}$. The experiments show a smaller dimensionless centerline film thickness towards the end whereas the values are significantly higher in the simulations. Since the crown in the experiment breaks into droplets, it acts like an elastic membrane for a longer time, all the liquid in the crown falls back into the center of the cavity, forcing it to fill more quickly in the experiment. 


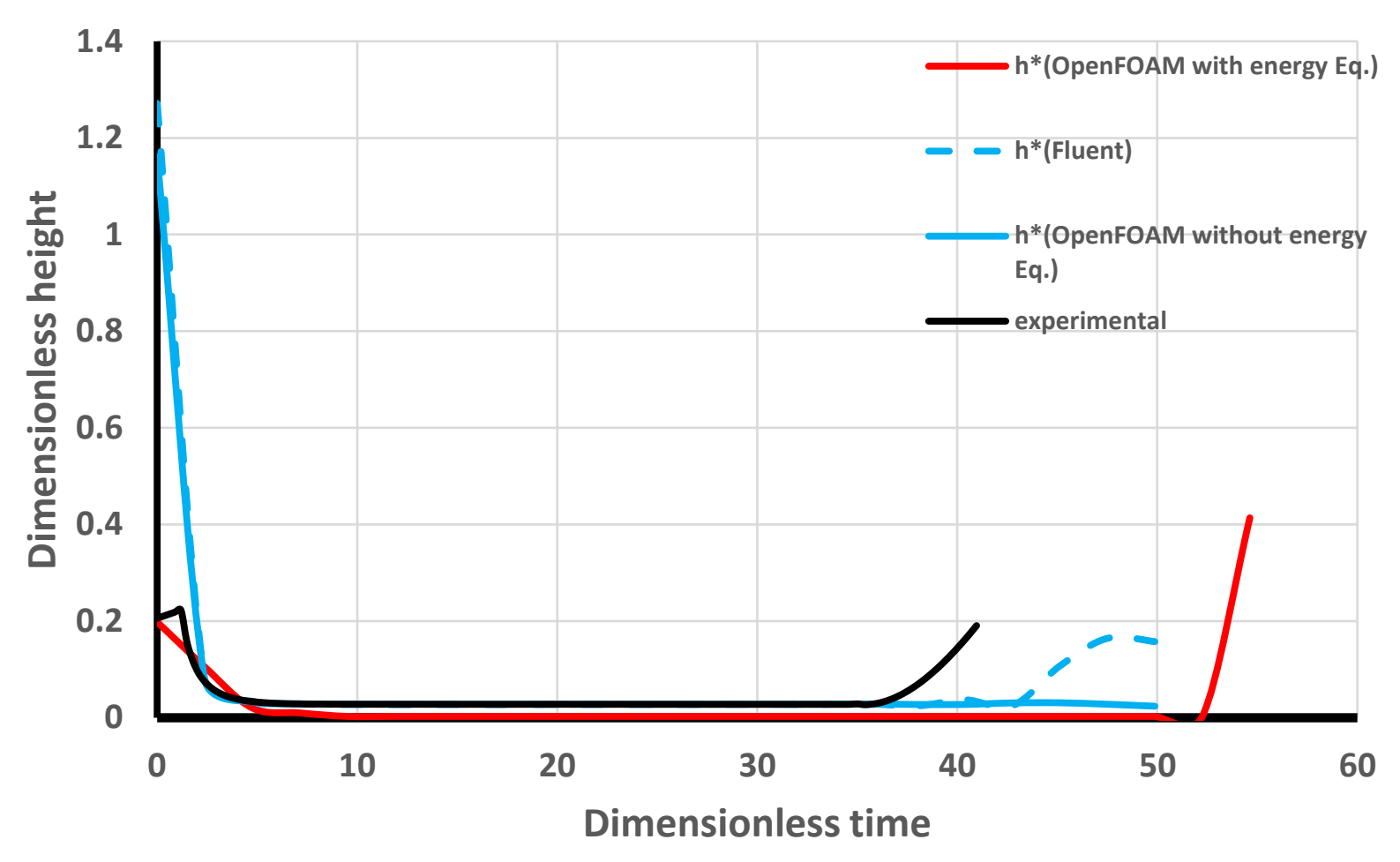

Fig 7.15 Centerline Film thickness for case $5 c(W e=984, R e=3555, h / d=0.2)$

\subsection{Comparison of Sub-cavity Volumes}

The method developed for the calculation of the sub-cavity volume has been discussed in Chapter 3. In this section, the comparison of the normalized sub-cavity volumes as a function of non-dimensional time of the CFD cases with the experiments is presented. The normalized sub-cavity volume is calculated by:

$$
V^{*}=\frac{V_{\text {sub-cavity }}}{V_{\text {drop }}}
$$

The non-dimensional time is again calculated as:

$$
t^{*}=t \frac{V e l}{d}
$$


Fig. 7.16 shows the comparison of normalized sub-cavity volume versus normalized time computed in different ways for the case $1 \mathrm{a}$. The value of sub-cavity volume is zero till the formation of cavity. It increases suddenly since the radius of the cavity rapidly increases. This case has the thickest liquid film with the lowest We. Low We cases don't splash. The Re is low due to a low velocity. Therefore most of the momentum generated due to the drop is spent in pushing the liquid away to form a cavity. The crown is barely formed, and cavity lifetime is short.

The rate at which the volume increases is almost the same in all the cases. As seen earlier, the cavity life times are different in CFD and experiments. This is believed to be because the experiment is performed in an environment that is not free from dust particles and temperature variations. The values of all the properties in CFD simulation are maintained constant. Hence, the difference.



Fig.7.16. Dimensionless volume vs Dimensionless time for case 1 a (We $=145, R e=1189, h / d=1)$ 
Fig.7.17 shows the variation of dimensionless sub-cavity volume as a function of dimensionless time for the case $1 \mathrm{c}$. The plots follow a similar trend as seen in previous case. But here the sub-cavity volume falls suddenly after the maximum radius of the cavity is reached for the CFD simulation.

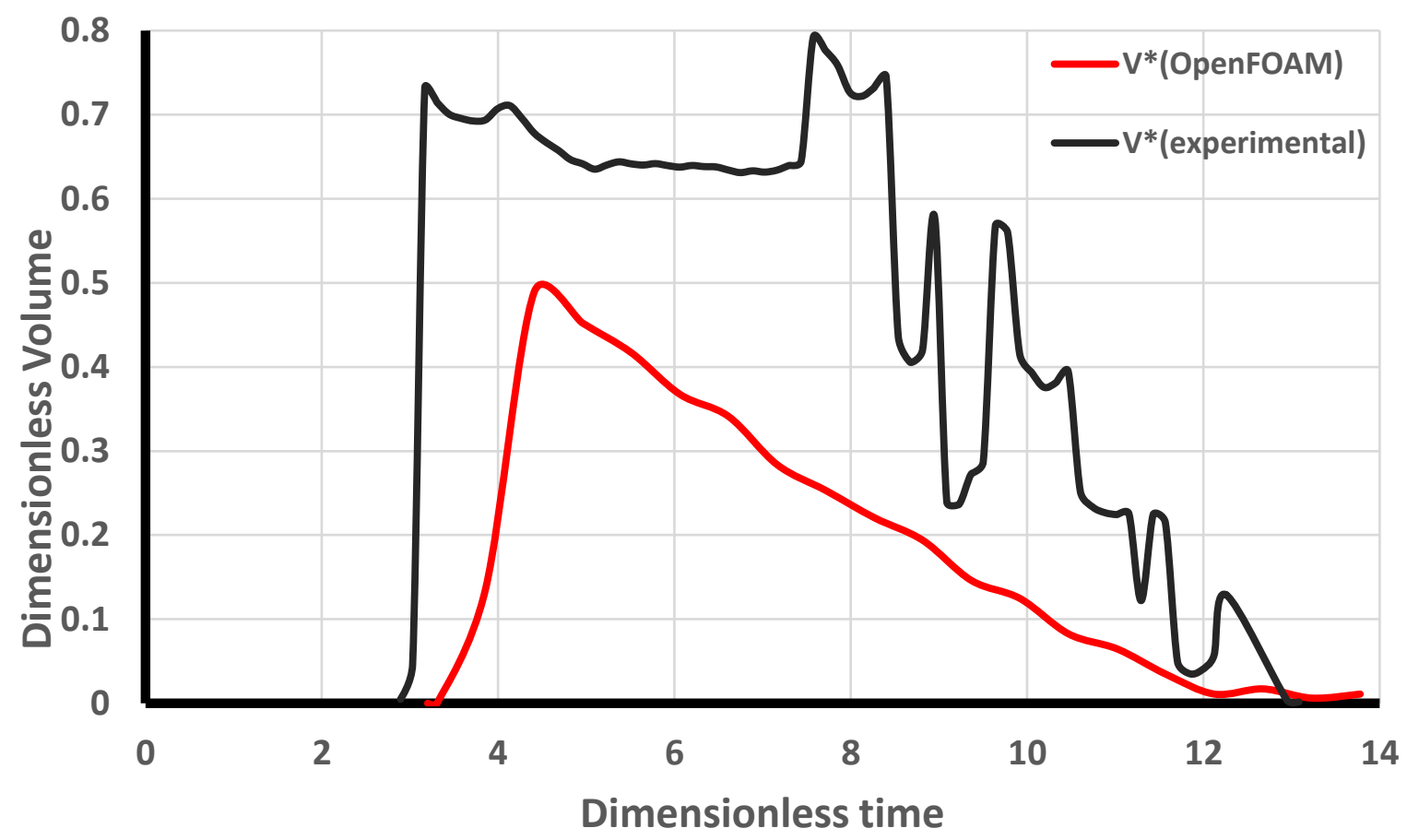

Fig.7.17. Dimensionless volume vs Dimensionless time for case $1 \mathrm{c}$ (We $=135, \operatorname{Re}=1176, \mathrm{~h} / \mathrm{d}$ $=0.2$ )

Fig.7.18 shows the comparison of normalized sub-cavity volumes as a function of normalized time for the CFD simulations with the experiment for the case $3 \mathrm{~b}$. This is an intermediate case between the highest We and the lowest We cases. The initial velocity of droplet impingement is larger than the first two cases. Therefore a larger cavity with increased cavity lifetime is observed. Despite some aberrations in the start, the CFD results show a good agreement with the experiments. The 
cavity fill-in process is slightly different for the experiments and the CFD simulations.

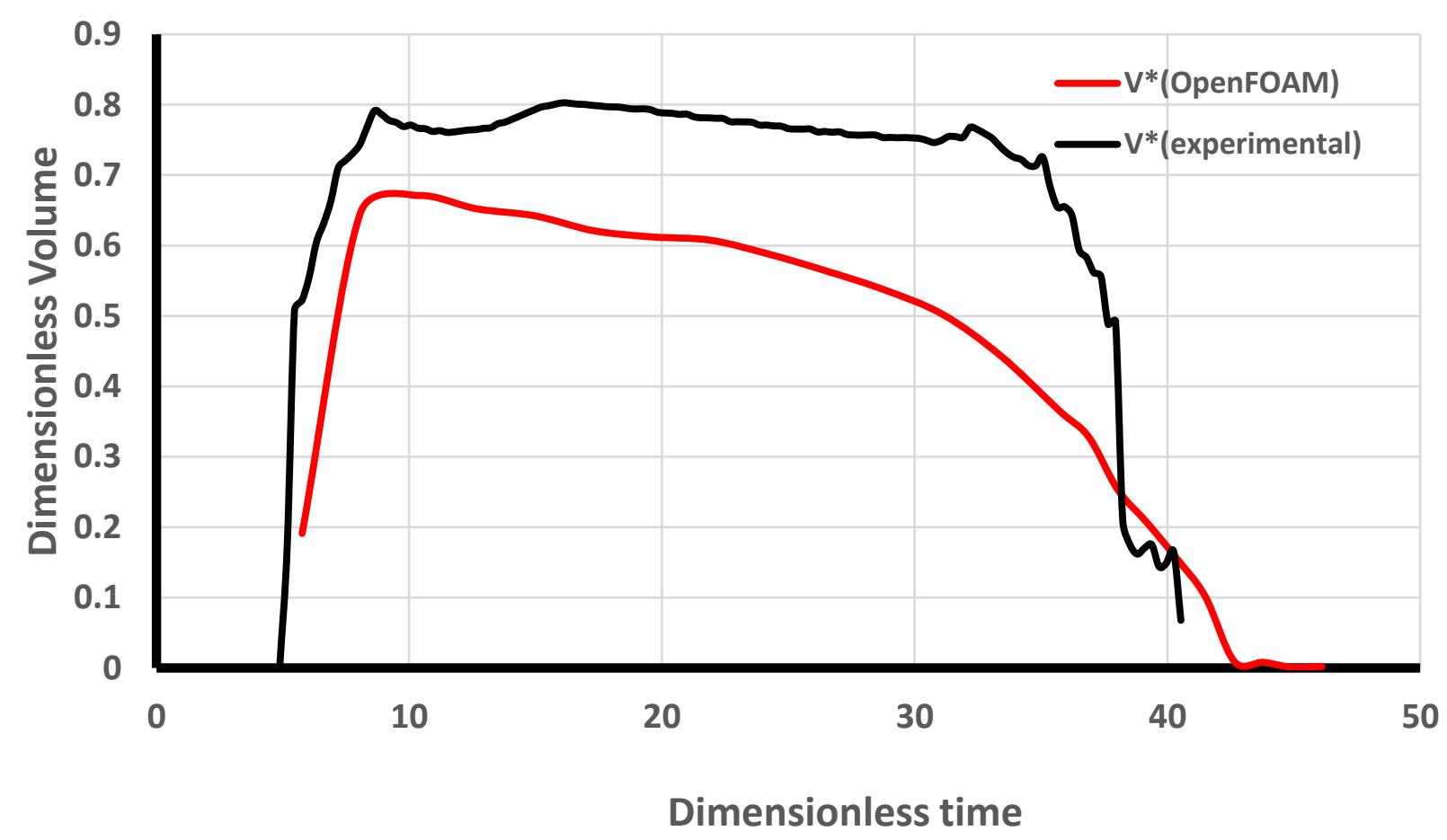

Fig.7.18 Dimensionless Volume vs Dimensionless Volume for case $3 b$ (We $=621, R e=2643$ $, h / d=0.5)$

The Fig.7.19 shows the plot of normalized sub-cavity volumes vs normalized time for the case 5 a. This case has the highest Weber number and the thickest liquid film. It also has the highest velocity, hence has the highest Reynolds number. Due to high velocity, the drop impacts the static liquid with large momentum and forms a cavity with even larger radius. Because of the momentum of the drop, there is 
also a steep rise in the $V^{*}$ curve in the initial stages of impact. This is also the primary reason for the longest cavity lifetime compared to the other cases.



Fig.7.19 Dimensionless Volume vs Dimensionless time for case 5 a (We $=993, R e=3574$, $h / d=0.9)$

Case $5 \mathrm{c}$ is different from the above cases and has high We and Re. Fig.7.19 shows the normalized sub-cavity volume curve vs the normalized time for the case $5 \mathrm{c}$. In addition to high $\mathrm{Re}$ and $\mathrm{We}$, this case has a very thin liquid film. Due to high momentum imparted to the thin liquid film, there are a lot of secondary splashing ejected out of the crown, which is very difficult to predict in the CFD simulations. As discussed earlier, the crown in this case acts like an elastic membrane so long, which expands due to the momentum of the drop and then contracts and falls back 
due to the surface tension. The cavity lifetime is effected greatly by this behavior and therefore the difference in results.

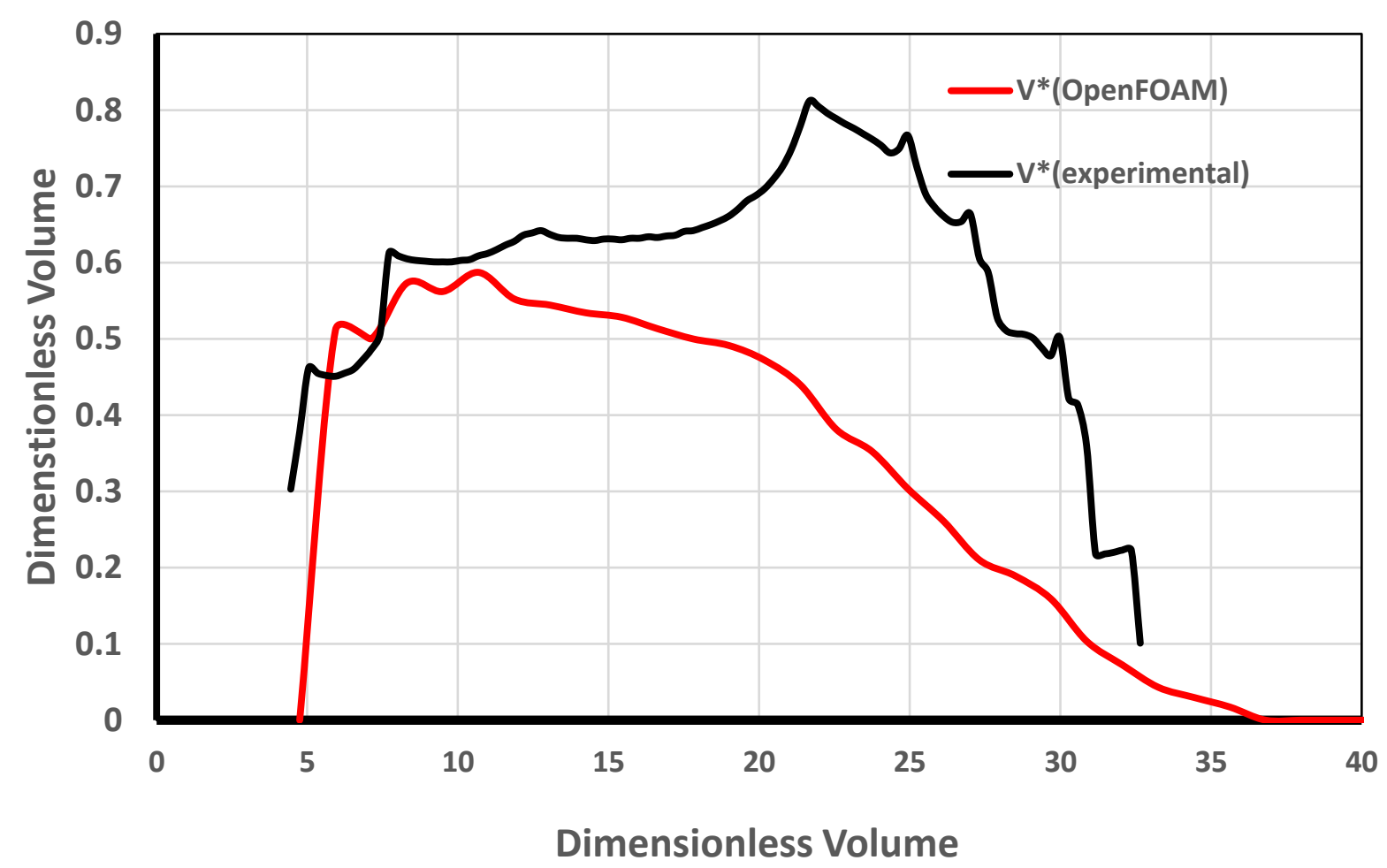

Fig.7.20 Dimensionless Volume vs Dimensionless time for case 5 c (We $=984, \operatorname{Re}=3555, h / d=0.2$ )

\subsection{Average heat flux}

As mentioned earlier, the average heat flux is calculated in a plane that is one mesh point above the bottom wall which is $372 \mathrm{~K}$. The process of calculating the heat flux has been explained earlier in the Chapter 6. Fig.7.21, Fig.7.22, Fig.7.23, Fig.7.24 and Fig 7.25 shows the plot of Average heat flux as a function of time for the cases 1 a, $1 \mathrm{c}, 3 \mathrm{~b}, 5$ a and $5 \mathrm{c}$, for both inside the cavity and outside the cavity. 


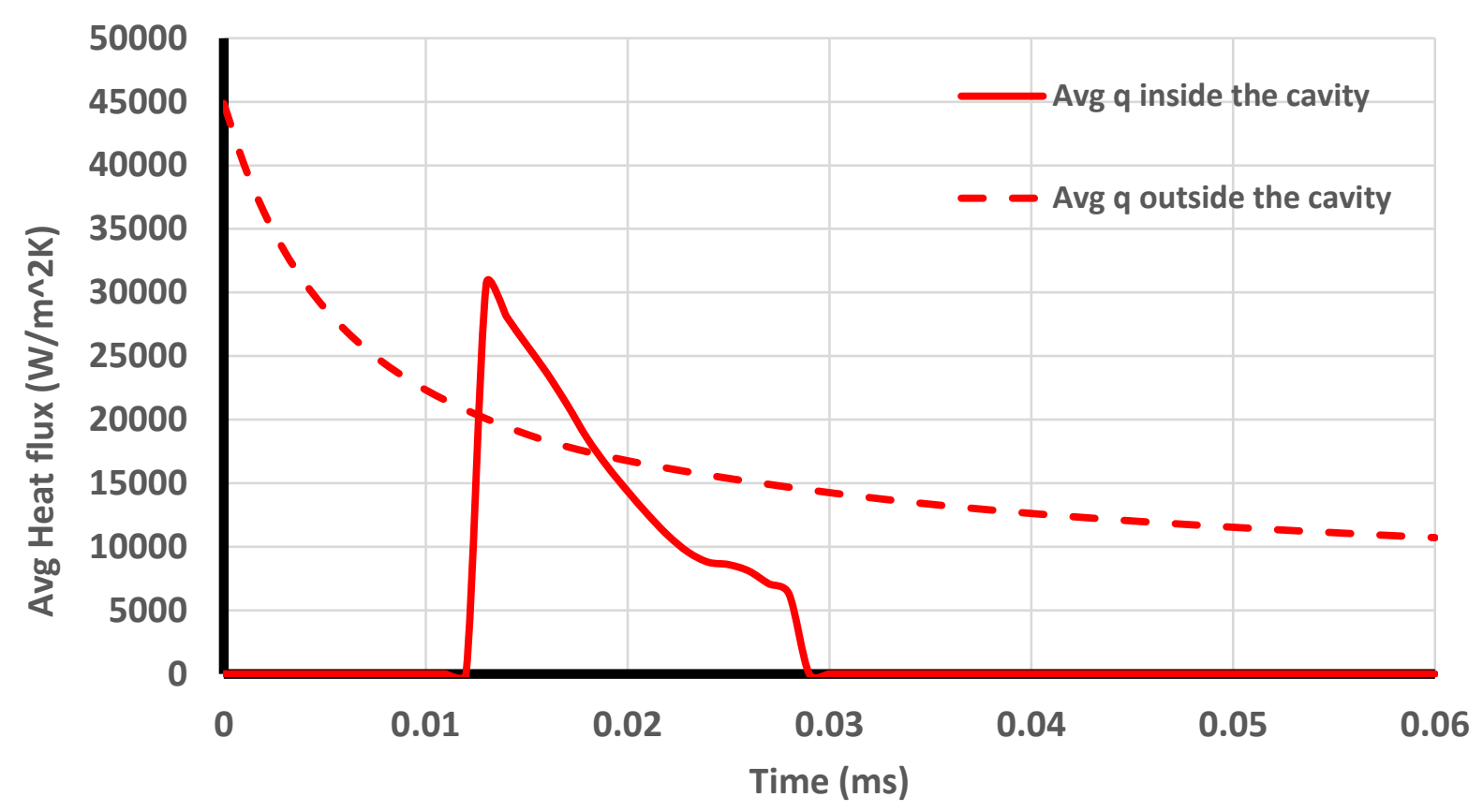

Fig.7.21 Avg heat fluxes inside and outside the cavity for the case 1 a (We=145, $R e=1176, h / d=1)$



Fig.7.22 Avg heat fluxes inside and outside the cavity for the case $1 \mathrm{c}(\mathrm{We}=135, \mathrm{Re}=1176, \mathrm{~h} / \mathrm{d}=$ 0.2) 




Fig.7.23 Avg heat fluxes inside and outside the cavity for the case $3 b(W e=621, R e=2643, h / d=$ 0.5)

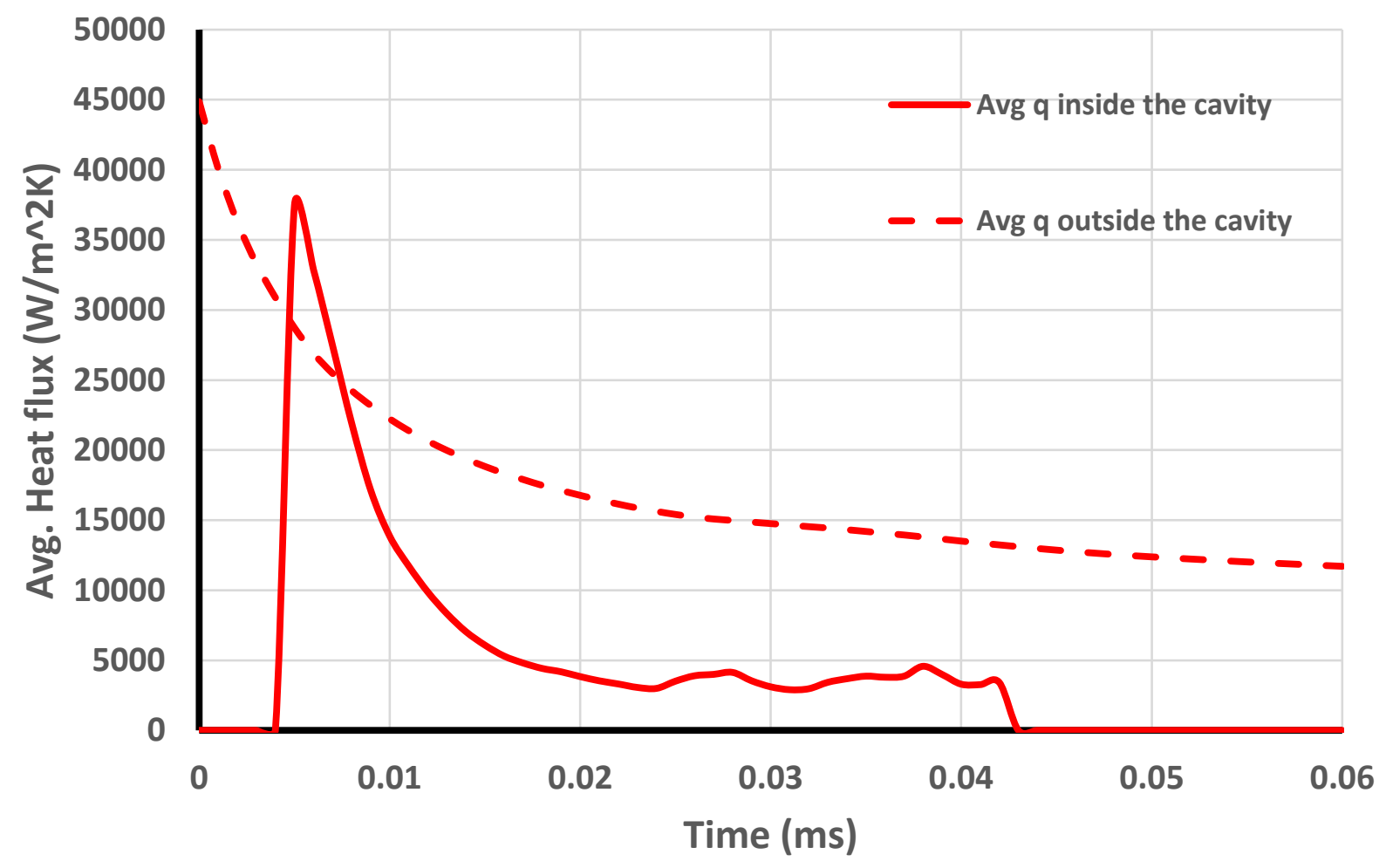


Fig.7.24 Avg heat fluxes inside and outside the cavity for the case 5 a (We=993, Re= $3574, h / d=0.9$ )

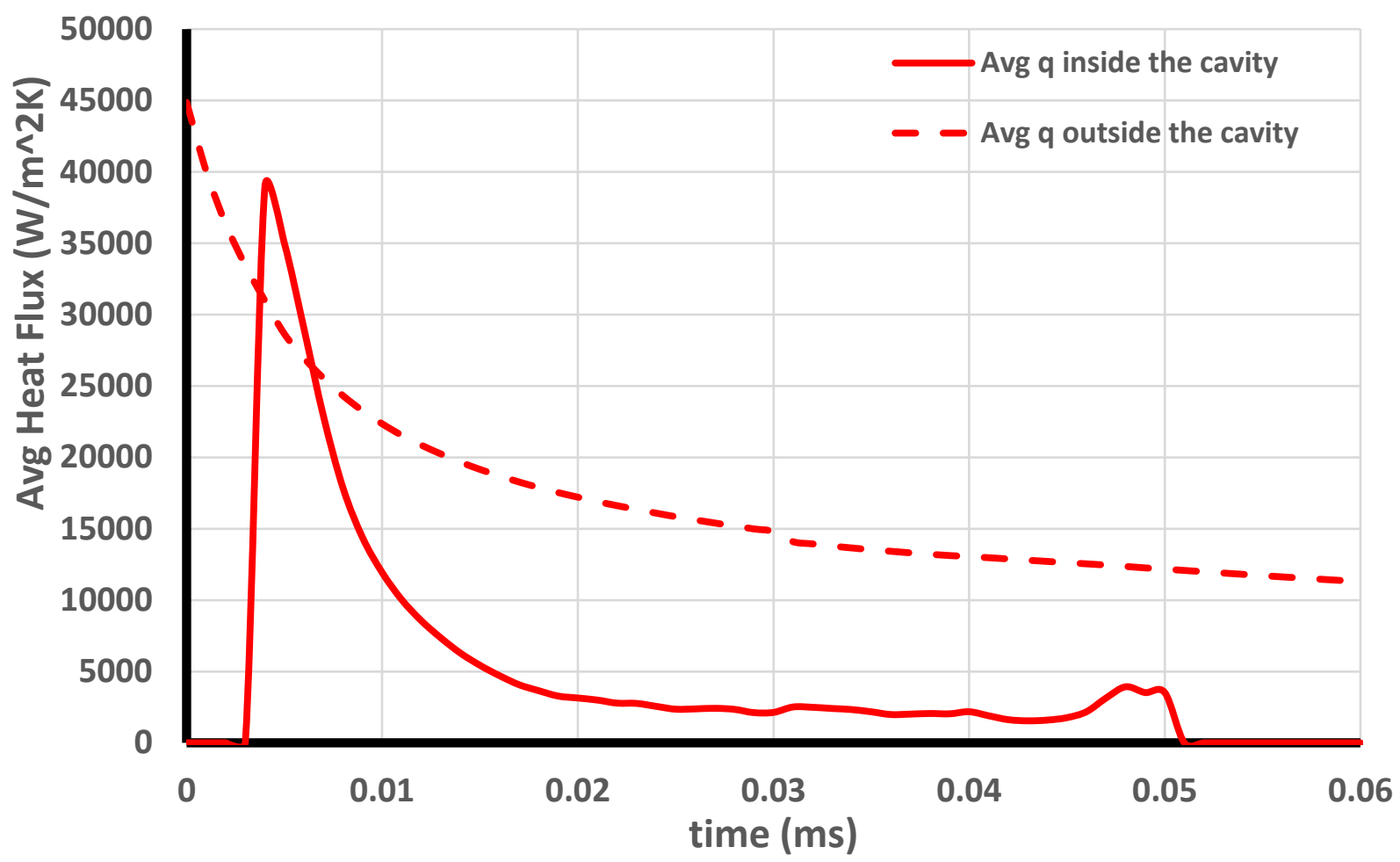

Fig.7.25 Avg heat fluxes inside and outside the cavity for case $5 \mathrm{c}$ (We $=984, \operatorname{Re}=3555, \mathrm{~h} / \mathrm{d}=$ 0.2)

The average heat flux under the cavity in the plots initially show zero because the cavity doesn't exist. The values under the cavity are sorted by sorting out all the values of alpha under the value of 0.9 . The introduction of the cavity causes the temperature to fall much quickly during the formation of the cavity, which is evident from all the graphs above.

The lowest heat flux in the sub-cavity is seen in case $1 \mathrm{a}$, where the Weber number is the lowest and the thickness of the film is the highest. It is high in the case $3 \mathrm{~b}$ where the thickness is more than the other two cases. Cases $1 \mathrm{c}$ and $5 \mathrm{c}$ have even higher peak in the heat flux in the sub-cavity. The heat flux outside the sub-cavity is low in the case of $3 \mathrm{~b}$ where the liquid film thickness is low when compared to 
other two cases. The heat flux predictions are worse in the case 5a and lower than the other two cases when seen in the later times.

The decrease in slopes as the Re and We increases is clearly seen. That means it takes lesser time for the cases with higher Re and We to cool down. The slope would be a constant had there been no droplet at all. Therefore spray cooling definitely showed improvement. All the plots almost start and end a same point because the dominance of the points outside the cavity which are almost at the same temperature for all the cases. 


\section{Conclusions and Future Work}

\subsection{Conclusions}

After studying and discussing the history and the application of spray cooling, it can be concluded that spray cooling is essential to achieve higher heat fluxes. Sprays have millions of droplets per second and only one of them was considered in this study. So the effect of spray cooling in the present study will be enhanced a lot when a spray is used. By varying the non-dimensional parameters (Re, We, $\left.h^{*}\right)$ of the liquid, the single drop impacts studied previously through experiments (Hillen, 2013) and through CFD simulations, both 2D and 3D (Medam, 2015) but both without the energy equation, and these results were compared to the present simulations.

Chapter 7 shows the results and in general, the simulations have good agreement with the experiments in the initial stages of the crown formation. Also, differences in the cavity life times can be seen. The CFD simulations slightly differ with some of the experimental results because the CFD simulations can't precisely take splashing into account whereas splashing occurs in some of the experiments. This is believed to primarily be due to the insufficient mesh refinement near the crown.

As mentioned earlier, the centerline film thickness time history comparisons of all the five cases between the experiments and the CFD simulations shows good agreement with the experiments in the early times. The retraction phase is not predicted well in CFD as the parameters remain constant in the simulation but vary in the experiments. Also, the limitations in the mesh would influence the predicted centerline film thickness at later times. 
The best agreement of experiments with the CFD simulations for the subcavity liquid volume is seen in the cases with higher We and Re. In cases with low $\mathrm{h} / \mathrm{d}$ value, the present simulations give a bad prediction of cavity lifetime and the behavior of crown.

The slope of the plot of average heat flux values decreases as the We and $\mathrm{Re}$ is increased. The $h / d$ value didn't have a much effect maybe because the time interval is too small. All the graphs look similar because of the dominant effect of the temperatures outside the cavity which are pretty much the same in all the cases at least in the earlier parts of the simulations.

\subsection{Future Work}

The present work is very useful in predicting the type of liquid and spray conditions to be used for the future study of the sprays. The predictions can be more accurate if a finer mesh is used. The mesh couldn't be finer in this work because of the restrictions on the performance of the computation. An adaptive mesh can improve and predict the results more accurately. Also, the present simulations should be re-analyzed in order to compute the percentages of sub-cavity volumes that originate from the drop liquid and the liquid layer.

Same work can be done with cylindrical or spherical domains to eliminate the problems that arise at the diagonal corners of the present rectangular mesh.

The heat flux predictions are done without considering the natural convection between the liquid surface and air. At infinite time, the profile would be a straight line at $372 \mathrm{~K}$ if done so. Also, if the temperature of the bottom wall is 
above $372 \mathrm{~K}$, phase change takes place, which changes the heat flux. That can also be considered for further simulations.

Simulations should be performed with different temperature profiles. It would be more realistic if the initial temperature in the static liquid layer varies in the vertical direction. The temperature of the liquid layer can't be the same throughout and the temperature of the component doesn't remain the same, as well. This could be the most important work that is yet to be undertaken. 


\section{References}

A.L.N. Moreira, A.S. Moita, M.R. Panao. 2010. "Advances and challenges in explaining fuel spray impingement: How much of single droplet impact research is useful?" Progress in Energy and Combustion Science.

Brian L. Rowden, R. Panneer Selvam, Eric A. Silk. 2006. "Spray Cooling Development Effort for Microgravity Environments." Proceedings of American Institute of Physics Conference. Proc. 813, 134,Alberquerque, New Mexico,Feb. 12-16,2006.

C.W.Hirt, B.D. Nicholas. 1981. "Volume of Fluid (VOF) method for the dynamics of free boundaries." Journal of Computational Physics 39.1: 201-205.

Dinc, Murat. 2015. "Computational Analysis of Single Drops and Sprays for Spray Cooling Applications.", Ph. D. Dissertation, West Virginia University, Morgantown, WV.

E. Gehring, G. Soriano, Y.P.Lin,J.L. Alvarado, M.F. Trujillo. 2010. "Film Dynamics Relevant to Spr Cooling." In Advanced Computational Methods and Experiments in Heat Transfer XI. WIT Trans. Eng. Sci. 68: 313-324.

Fan Jiang, Yijun Wang, Jianhua Xiang, Zhenzhang Liu. 2010. "A Comprehensive Computational Fluid Dynamics Study of Droplet-Film impact and Heat Transfer ." Chemical Engineering Technology, june 10: 1565-1573.

Guao Yali, Wei Len, Shen Shenqiang, Chen Guiyang. 2014. Simulation of Dynamic Charecteristics of droplet impact on a liquid film. Dalian, China: School of Energy and Power Engineering, Dalian University of Technology.

Guillermo Soriano, Jorge L. Alvarado, Yen Po Lin,. 2010. "Experimenatal Characterization of single and multiple droplet impingement on surfaces subject to constant heat flux." Proceedings of the 14th Int'I. Heat Transfer Conference ITHC14, Washington, DC, Aug. 8-13, 2010.

Hillen, Nicholas Lee. 2013. "Droplet impact Sub-cavity histories and PDPA spray cooling modeling." Master's Thesis, West Virginia University, Morgantown, WV.

Jinlong Xie, Teck Neng Wong, Fei Duan. 2016. "Modelling on the dynamics of droplet impingement and bubble boiling in spray cooling." International Journal of Thermal Sciences 104: 469-479.

Joseph E. Johnston, R.P. Selvam, Eric A. Silk. 2005. "Spray Cooling Modelling: Droplet Sub-Cooling Effect on Heat Transfer." Proceedings of Space Technologies and International Forum (STAIF) Conference, Jan 2008

Kreitzer, P.J. 2010. "Spray Cooling simulation implementing time scale analysis and Monte Carlo method." Ph. D. Dissertation, West Virginia University, Morgantown, WV.

Kyriopoulos, Olympia Natalia. 2010. "Gravity effect on liquid film hydrodynamics and spray cooling." Ph. D. Dissertation, Darmstardt Technical University, Darmstardt, Germany.

Medam, Krishna Teja. 2015. "Comparisons of Single Drop Impact Simulations with Experiments." Morgantown, WV: Masters Thesis, West Virginia University. 
Nicholas Lee Hillen, John M. Kuhlman, Murat Dinc, Donald D. Gray. June 25-28, 2012. "Droplet impingement on wet and dry surfaces." AIAA 42nd Fluid Dynamics Conferences. New Orleans, LA.

R. Panneer Selvam, Matthew Hamilton, Eric A. Silk. 2006. "Spray Cooling Modelling: Liquid Film Thickness Effect on Heat Transfer."Proceedings of American Institute of Physics Conference Processings 813. 134, Abuqyurque, New Mexico, Feb. 12-16

R. Panneer Selvam, Mita Sarkar, Suranjan Sarkar, Rengasamy Ponnappan. 2007. "Effect of Vapor Bubble Size on Heat Transfer in Spray Cooling."STAIF $11^{\text {th }}$ Conference on Thermodynamics Applications in Microgravity, Albuquerque, New Mexico, Feb. 11-15

Suranjan Sarkar, R.Panneer Selvam. 2009. Direct Numerical Simulation of Heat Transfer in Multiphase Flow Modeling Using Parallel Computing. Computational Mechanics Laboratory. University of Arkansas, Fayetteville, AR.

Xiaoyang Xu, Jie Ouyang, Tao Jiang. 2014. "Numerical analysis of the impact of two droplets with a liquid film using an incompressible SPH method."Journal of Mathematics 85:35-53 